\title{
Environmental Dynamical Control of Tropical Cyclone Intensity- An Observational Study
}

\author{
ZHIHUA ZENG* \\ International Pacific Research Center, and Department of Meteorology, School of Ocean and Earth Science and Technology, \\ University of Hawaii at Manoa, Honolulu, Hawaii, and Shanghai Typhoon Institute, Shanghai, China \\ YUQING WANG \\ International Pacific Research Center, and Department of Meteorology, School of Ocean and Earth Science and Technology, \\ University of Hawaii at Manoa, Honolulu, Hawaii \\ Chun-Chieh Wu \\ Department of Atmospheric Sciences, National Taiwan University, Taipei, Taiwan
}

(Manuscript received 9 January 2006, in final form 1 April 2006)

\begin{abstract}
The effects of two environmental dynamical factors, namely, the transitional speed and vertical wind shear, on tropical cyclone (TC) intensification, intensity, and lifetime peak intensity were analyzed based on observations in the western North Pacific during 1981-2003. In general, both the fast translation and strong vertical shear are negative to both TC intensification and the lifetime peak intensity. Both the very intense TCs and the TCs with rapid intensification rate are found only to occur in a narrow range of translational speeds between 3 and $8 \mathrm{~m} \mathrm{~s}^{-1}$, and in relatively weak vertical shear. The overwhelming majority of western North Pacific TCs reach their lifetime peak intensity just prior to recurvature where their environmental steering flow and vertical shear are both weak. The results show that few TCs intensified when they moved faster than $15 \mathrm{~m} \mathrm{~s}^{-1}$, or when their large-scale environmental vertical shear is larger than $20 \mathrm{~m} \mathrm{~s}^{-1}$. The intensification rate of TCs is found to increase with decreasing vertical shear while the majority of the weakening storms experience relatively strong vertical shear. Overall, strong vertical shear prohibits rapid intensification and most likely results in the weakening of TCs, similar to the fast storm translation. Based on the statistical analysis, a new empirical maximum potential intensity (MPI) has been developed, which includes the combined negative effect of translational speed and vertical shear as the environmental dynamical control in addition to the positive contribution of SST and the outflow temperature as the thermodynamic control. The new empirical MPI can not only provide more accurate estimation of TC maximum intensity but also better explain the observed behavior of the TC maximum intensity and help explain the thermodynamic and environmental dynamical controls of TC intensity. Implications of the new empirical MPI are discussed.
\end{abstract}

\section{Introduction}

The intensity of a tropical cyclone (TC), measured as the maximum sustained surface wind or minimum cen-

\footnotetext{
* Additional affiliation: Shanghai Typhoon Institute, Shanghai, China.
}

Corresponding author address: Dr. Yuqing Wang, IPRC/ SOEST, University of Hawaii at Manoa, 1680 East-West Rd., POST Bldg. Room 409G, Honolulu, HI 96822.

E-mail: yuqing@hawaii.edu

DOI: 10.1175/MWR3278.1

(C) 2007 American Meteorological Society tral pressure, is affected at any time by an array of complex physical processes that govern the interaction of the TC both with the underlying ocean and with its atmospheric environment. The lack of a deep understanding of the physical processes/mechanisms critical to TC intensity is often attributed to the low skill in current intensity forecast (Emanuel 2000). The intensity of a TC can be considered as being controlled by both dynamical and thermodynamic processes. We refer to them as dynamical control and thermodynamic control, respectively. 


\section{a. Thermodynamic control}

The sea surface temperature (SST) and the atmospheric thermodynamic structure (such as the uppertropospheric temperature) are regarded as the thermodynamic controls of TC intensity. Observational studies have shown that SST is a significant determinant of TC intensity. Palmén (1948) found that TCs in the Northern Hemisphere formed over oceans with SST higher than $26^{\circ}-27^{\circ} \mathrm{C}$. Gray $(1968,1978)$ emphasized that the $26^{\circ} \mathrm{C}$ isotherm extending down to a depth of $60 \mathrm{~m}$ from the surface is required for the TC to develop.

SST determines the amount of sensible and latent heat available to the TC from the ocean and, thus, is indicative of the potential TC intensity (Miller 1958; Malkus and Riehl 1960). This enabled Merrill (1988) to obtain an empirical maximum TC intensity for a given SST. Emanuel (1986), on the other hand, derived a theoretical upper bound of intensity, namely the maximum potential intensity (MPI) that a TC can achieve given both the underlying SST and the atmospheric thermodynamic environment conditions. By this theory, a TC is regarded as an ideal Carnot heat engine gaining energy from the disequilibrium between the atmospheric boundary layer and the ocean surface and losing energy in the upper-level outflow layer. This airsea interaction theory of the TC intensity has been further elaborated subsequently by Emanuel (1988, 1991, 1995). With a different approach, Holland (1997) also described a theoretical MPI, which is solely determined by the thermodynamic conditions of the ocean and the atmospheric environment.

The MPI theories only take into account the positive feedback between the TC and the underlying ocean. In a coupled hurricane-ocean model, Bender et al. (1993) showed that the ocean cooling due to strong turbulent mixing provides an important negative feedback to TC intensity. With an interactive ocean in a simple axisymmetric TC model, Emanuel (1999) successfully simulated the intensification of real TCs and indicated that thermodynamic control plays a dominant role in most cases. One of the caveats in Emanuel's study was the difficulty in simulating the development of storms embedded in environmental flow with vertical shear, indicating the importance of dynamical control of TC intensity.

\section{b. Dynamical control}

Dynamical control of TC intensity is much more complicated than the thermodynamic control because of different dynamical processes involved. Based on the nature of the dynamics, dynamical control can be di- vided into internal dynamics and environmental dynamical forcing (Wang and Wu 2004). Internal dynamics include the eyewall process, the interaction between the eyewall and spiral rainbands, and the vortex Rossby wave dynamics in the eyewall and the embedded mesovortices (Willoughby et al. 1982; Montgomery and Kallenbach 1997; Montgomery and Enagonio 1998; Wang 2001, 2002a,b; Camp and Montgomery 2001; Wang and $\mathrm{Wu}$ 2004). The environmental forcing may include the so-called beta effect, uniform environmental flow, vertical shear of horizontal wind, and interactions with another TC or an upper-tropospheric trough (Gray 1968; Merrill 1988; Wang and Holland 1995; Peng et al. 1999; Titley and Elsberry 2000; Hanley et al. 2001).

The effect of internal dynamics on TC intensity has been increasingly understood in the past decade or so. Based on the barotropic vorticity dynamics argument, Montgomery and Kallenbach (1997) and Montgomery and Enagonio (1998) suggested that vortex Rossby waves might contribute to TC intensification through axisymmetrization process. This was further demonstrated in more sophisticated dynamical frameworks by Möller and Montgomery (1999, 2000). In a recent study, Yang et al. (2007) show that the vortex Rossby waves in the inner core of a TC could reduce the maximum intensity by $15 \%$. Therefore, the internal wave dynamics may play different roles at different stages of a TC. Although it is not clear whether the development of a concentric eyewall is an internal dynamical process or involves large-scale forcing (Willoughby et al. 1982, 1984; Nong and Emanuel 2003); when developed, it is a major process that can cause large intensity fluctuation of a TC and may terminate the further intensification in many cases (Camp and Montgomery 2001). Another important process that can be regarded as internal dynamics is the complex interaction between the eyewall and spiral rainbands. Wang (2002b) showed that strong spiral rainbands could provide an asymmetric forcing to the eyewall convection, leading to the eyewall breakdown and partial eyewall replacement, accompanied by an intensity oscillation. In addition, cloud microphysical processes may also be regarded as internal dynamics, which may affect both the evolution and intensity of TCs (Wang 2002c). Although internal dynamics play important role in affecting TC intensity change and in determining the final TC maximum intensity, it is still not conclusive and needs further detailed investigation, including observational studies.

In addition to the internal dynamics, external forcing from large-scale environmental flow, such as the vertical shear, is a key factor affecting TC intensity (Wu and Cheng 1999; Emanuel et al. 2004; Wang and Wu 2004). 
Such external forcing is usually regarded as environmental dynamical control of TC intensity (Emanuel 2000). Among different kinds of external forcing, the vertical shear has been long known to be detrimental to TC genesis and intensification (Gray 1968; McBride and Zehr 1981; Merrill 1988). Zehr (1992) determined a threshold value for vertical shear of $12.5 \mathrm{~m} \mathrm{~s}^{-1}$ above which TCs could not form in the western North Pacific. An early explanation of vertical shear effect is the socalled "ventilation" effect (Gray 1968), namely, advection of warm core by flow in the upper levels relative to the low-level TC circulation. An alternative explanation of the ventilation effect is the outward eddy flux of warm core air by the shear-induced asymmetric flow in the upper levels, causing an up-down decrease of the warm core and thus weakening of the TC in vertical shear (Frank and Ritchie 2001). Although vertical shear can inhibit TC genesis and intensification, the well-developed, large TCs may resist relatively quite strong vertical shear (DeMaria 1996; Wang et al. 2004; Wong and Chan 2004). By introducing several environmental effects in his simple coupled axisymmetric TC model, Emanuel et al. (2004) recognized that the greatest source of uncertainty in forecasts of TC intensity is uncertainty in forecast values of the environmental vertical shear.

Another issue related to the vertical shear effect is the upper-tropospheric trough from the midlatitude. In addition to introducing vertical shear to a TC, the upper-level trough also imposes a cyclonic PV anomaly and in some cases may contribute to TC intensification (Titley and Elsberry 2000). Hanley et al. (2001) examined 121 Atlantic TCs in an attempt to differentiate between troughs that lead to intensification (good trough), and those that lead to decay (bad trough). They found that a larger and stronger upper PV anomaly (bad trough) induces more vertical shear than a small-scale PV anomaly (good trough), and has a negative impact on TC intensity. Therefore, the "bad trough" or "good trough" for TC intensity likely depends on how the TC core responds to the forcing from the upper-level PV anomaly of different scales.

Compared with the effect of vertical shear and upper-level troughs, the effect of uniform environmental flow on TC intensification and intensity has received less attention. In their numerical experiments, Peng et al. (1999) found a reduction of the intensity for a TC embedded in a uniform environmental flow. They showed that the TC intensity was inversely proportional to the magnitude of the wavenumber- 1 asymmetry, which in turn was forced by asymmetric friction due to the imposed environmental flow (Shapiro 1983; Kepert 2001; Kepert and Wang 2001). Peng et al. (1999) indicated that an out-of-phase condition between the asymmetric surface entropy flux and boundary layer moisture convergence could explain the intensity reduction. Dengler and Keyser (2000) found that, in their three-layer model, it was the penetration of stable dry air from midlevels into the regions of boundary layer convergence that reduced the intensity of the storm embedded in uniform environmental flows. Recently $\mathrm{Wu}$ and Braun (2004) suggested that the inhibiting effect of environmental flow is closely associated with the resulting eddy momentum flux, which tends to decelerate tangential and radial winds in both inflow and outflow layers. Regardless of the physical processes, these numerical results show that uniform environmental flow or the environmental steering effect could be a limiting factor to TC intensification and intensity. Such an effect has been seen from observations in the Australian region as shown by Holland (1997) and Wang and $\mathrm{Wu}$ (2004), where there was a clear trend toward weaker TCs as the translational speed increases.

\section{c. The objectives of this study}

As discussed above, the thermodynamic control of TC intensity has been well established. This is reflected in both theoretical and empirical MPI of TCs (Miller 1958; Emanuel 1986; Holland 1997). The statistical analysis of Atlantic TCs by DeMaria and Kaplan (1994b) revealed that most storms could only reach $55 \%$ of their thermodynamic MPIs and only about $20 \%$ reach $80 \%$ or more of their MPIs at the time when they are most intense. Despite the fact that the current MPI theories have proven to be very good at estimating the MPIs of real TCs (Tonkin et al. 2000; Free et al. 2004), they have not included any dynamical control, such as the environmental flow and vertical shear. Since the dynamical control could be a limiting factor to TC intensity, a question arises as to whether its effect can be studied from observations and included in the empirical MPI estimation.

In fact, many dynamical factors affecting TC intensity, such as the storm translation and vertical shear, are considered as predictors in current operational statistical TC intensity prediction models, such as the Statistical Hurricane Intensity Prediction Scheme (SHIPS; DeMaria and Kaplan 1994a, 1999; DeMaria et al. 2005), and the Statistical Typhoon Intensity Prediction Scheme (STIPS; Knaff et al. 2005). However, a detailed analysis of these predictors has been lacking. Such an analysis will undoubtedly provide insight into the physics of dynamical control of TC intensity.

The objectives of this study are twofold: 1) we will first undertake an analysis of the effects of translation and vertical shear on TC intensity, respectively, from 
observations over the western North Pacific; 2) we will then construct a new empirical MPI for TCs in the region that includes not only the thermodynamic control of SST and outflow temperature but also the effects of storm translation and vertical shear of the environmental flow. The latter may provide additional guidance to the TC MPI estimation for intensity forecasts.

The next section describes the datasets. Section 3 briefly discusses control of TC intensity by SST over the western North Pacific and constructs an empirical MPI as a function of SST. The effect of TC translation on TC intensity and intensity change will be analyzed in section 4. The effect of vertical shear on TC intensity and intensity change will be the topic of section 5. In section 6, a new empirical MPI incorporating explicitly both the thermodynamic control and the environmental dynamical control will be developed based on the observations. Main conclusions will be drawn in the last section.

\section{Datasets and analysis methods}

The principal parameters/fields examined in this study include TC position and intensity, SST, vertical shear of the large-scale environmental flow, and the temperature near the tropopause. The analysis is restricted to the period from 1981 to 2003 over the western North Pacific. This period is chosen so that all the datasets are available to us. In addition, the best-track TC data for the region during this period was more consistent and reliable than pre-1981.

\section{a. Tropical cyclone best-track data}

The TC position and intensity information was obtained from the Joint Typhoon Warning Center (JTWC). The dataset is a postanalyzed product that contains additional information not available in the operational setting, allowing the best determination of TC position and intensity, the so-called best track (available online at http://weather.unisys.com/hurricane/ w_pacific/index.html). The data contain 6-hourly TC latitude, longitude, and maximum sustained surface wind for all TCs designated by JTWC as being tropical storm strength with actual maximum surface wind greater than $17 \mathrm{~m} \mathrm{~s}^{-1}$. To assure a homogeneous analysis, only TCs over the ocean without significant effects by landfall were included in our analysis, namely within the region of $0^{\circ}-50^{\circ} \mathrm{N}, 110^{\circ} \mathrm{E}-170^{\circ} \mathrm{W}$.

\section{b. SST}

The SST data used are the Reynolds SST reanalysis provided by the National Oceanic and Atmospheric
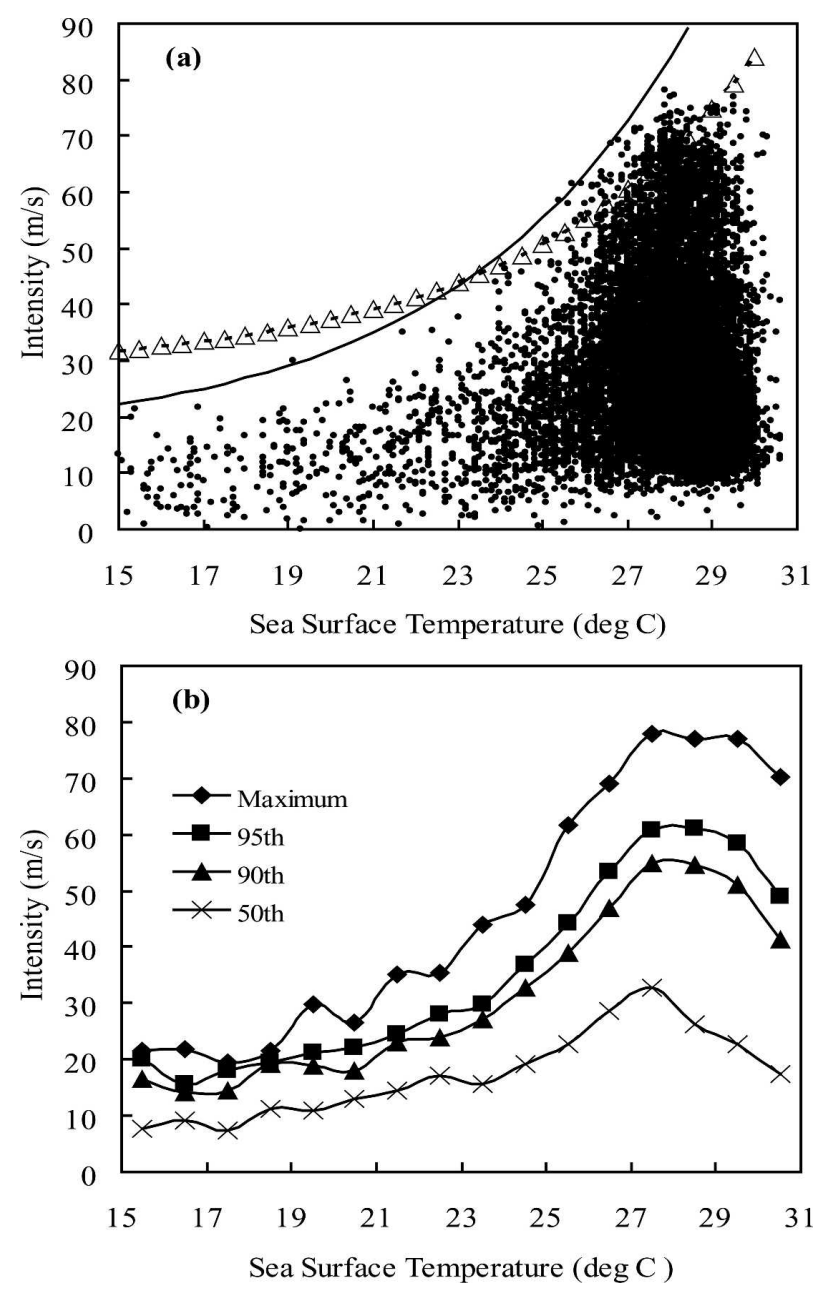

FIG. 1. (a) Scatter diagram of TC intensity (maximum surface sustained wind in $\mathrm{m} \mathrm{s}^{-1}$ ) vs SST $\left({ }^{\circ} \mathrm{C}\right)$ over the western North Pacific during 1981-2003. The intensity was corrected by subtracting the storm translational speed. The empirical MPI $\left(\mathrm{m} \mathrm{s}^{-1}\right)$ as a function of SST $\left({ }^{\circ} \mathrm{C}\right)$ derived for the western North Pacific is shown by the solid curve. The dashed curve with triangles in (a) shows the empirical MPI for Atlantic from DeMaria and Kaplan (1994b). (b) The maximum intensity and the 95th, 90th, and 50th intensity percentiles for each $1^{\circ} \mathrm{C}$ SST group as defined in DeMaria and Kaplan (1994).

Administration (NOAA) Cooperative Institute for Research in Environmental Sciences (CIRES) Climate Diagnostics Center (CDC) and can be obtained online at http://www.cdc.noaa.gov. The Reynolds SST is a weekly mean with a horizontal resolution of $1^{\circ}$ latitude-longitude (Reynolds et al. 2002).

\section{c. Wind and temperature reanalysis data}

The National Centers for Environmental PredictionNational Center for Atmospheric Research (NCEPNCAR) reanalysis products (Kalnay et al. 1996) were 
TABLE 1. Properties of the SST groups.

\begin{tabular}{cccc}
\hline \hline $\begin{array}{c}\text { SST midpoint } \\
\left({ }^{\circ} \mathrm{C}\right)\end{array}$ & $\begin{array}{c}\text { No. of } \\
\text { observations }\end{array}$ & $\begin{array}{c}\text { Avg intensity } \\
\left(\mathrm{m} \mathrm{s}^{-1}\right)\end{array}$ & $\begin{array}{c}\text { Avg top 50\% } \\
\text { intensity }\left(\mathrm{m} \mathrm{s}^{-1}\right)\end{array}$ \\
\hline 15.50 & 17 & 9.70 & 13.69 \\
16.50 & 26 & 9.25 & 13.45 \\
17.50 & 18 & 9.23 & 14.37 \\
18.50 & 24 & 11.95 & 16.17 \\
19.50 & 36 & 12.23 & 16.73 \\
20.50 & 49 & 12.50 & 17.15 \\
21.50 & 56 & 14.51 & 19.62 \\
22.50 & 73 & 17.03 & 22.50 \\
23.50 & 123 & 16.65 & 23.28 \\
24.50 & 211 & 20.48 & 27.86 \\
25.50 & 464 & 24.42 & 32.81 \\
26.50 & 1601 & 29.88 & 40.25 \\
27.50 & 3113 & 34.03 & 46.35 \\
28.50 & 5126 & 30.20 & 42.13 \\
29.50 & 2209 & 27.06 & 38.15 \\
30.50 & 86 & 23.53 & 33.50 \\
\hline
\end{tabular}

utilized to estimate the vertical shear of large-scale environmental flow and the outflow temperature of a TC. The data are available at 4 times daily and have a horizontal resolution of $2.5^{\circ}$ latitude-longitude with 17 vertical levels.

\section{d. Calculations of translational speed, vertical shear, and outflow temperature}

The translational speed of a TC was calculated using the centered time differencing based on the observed changes in longitude and latitude at 6-h intervals, except for the first and the last records where one-sided time differencing was used.

In the literature, the vertical shear of large-scale environmental flow was defined as the difference of the area-averaged winds between 200 and $850 \mathrm{hPa}$. The area used in the average however varied in different studies. For example, Elsberry and Jeffries (1996) used an area within a radius of $3^{\circ}$ latitude, while Franklin et al. (1993) used a radius of $6^{\circ}$ latitude. In this study, the vertical shear is estimated from the NCEP-NCAR reanalysis and defined as the difference of winds between 200 and $850 \mathrm{hPa}$ averaged within a circle of $5^{\circ}$ latitude around the TC center. This estimation may be affected by the displacement of the TC circulation in the reanalysis. However, since such a displacement is not systematic, it is not expected to affect our analysis significantly.

The upper-tropospheric outflow temperature, $T_{\text {out }}$, is estimated in this study as the temperature averaged within a radius of $5^{\circ}$ latitude near the tropopause. This may result in a slightly too cold outflow temperature as defined in Emanuel $(1988,1995)$ but our results show
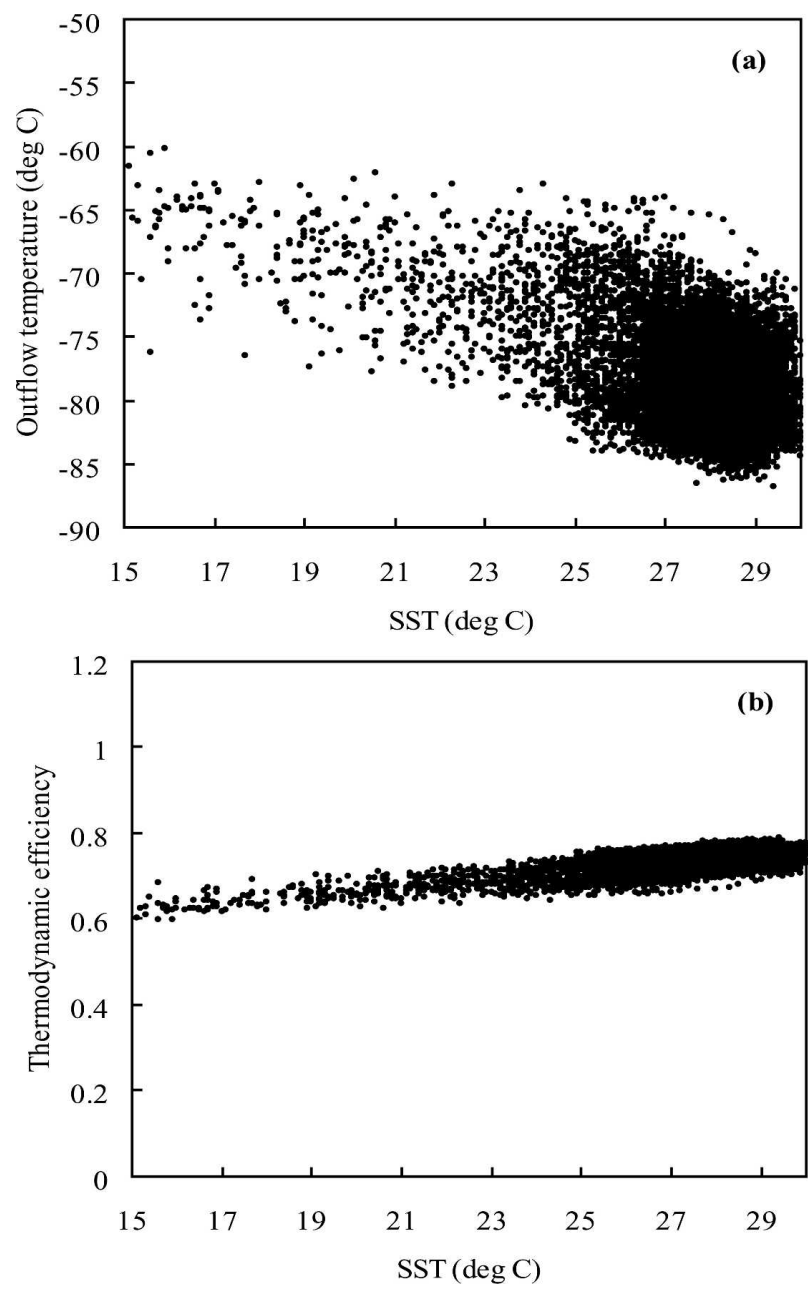

FIG. 2. (a) Scatter diagram of outflow temperature $\left({ }^{\circ} \mathrm{C}\right)$ vs SST $\left({ }^{\circ} \mathrm{C}\right)$ for western North Pacific TCs during 1981-2003. (b) The corresponding thermodynamic efficiency defined by Eq. (1).

that this is a quite reasonable estimation. As shown by Bister and Emanuel (1998), the theoretical maximum intensity measured by the near-surface wind speed is proportional to the thermodynamic efficiency defined as

$$
\varepsilon=\sqrt{\frac{\mathrm{SST}-T_{\text {out }}}{T_{\text {out }}}} .
$$

We will show that the outflow temperature is highly correlated to the underlying SST. As a result, $\varepsilon$ is determined largely by SST.

\section{TC intensity versus SST}

To isolate the dynamical and thermodynamic controls of TC intensity, we first examine the relationship between TC intensity and SST and construct an empirical TC MPI as a function of SST over the western North 

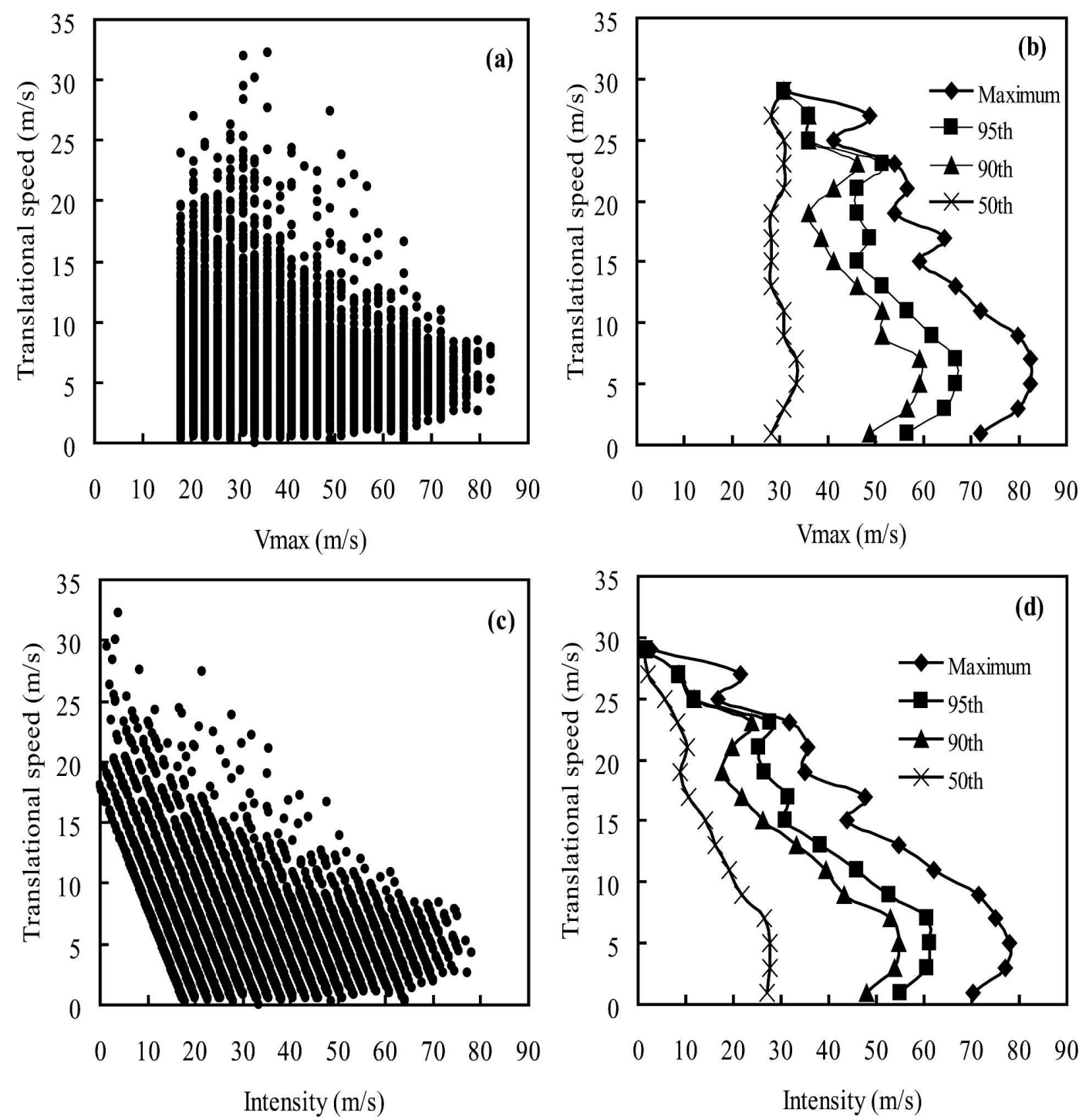

FIG. 3. Scatter diagrams of TC intensity $\left(\mathrm{m} \mathrm{s}^{-1}\right)$ vs translational speed $\left(\mathrm{m} \mathrm{s}^{-1}\right)$ from (a) the best-track intensity and (c) that corrected by subtracting storm translational speed over the western North Pacific during 1981-2003, and (b), (d) the corresponding maximum intensity and the 95th, 90th, and 50th intensity percentiles for each $2 \mathrm{~m} \mathrm{~s}^{-1}$ translational speed group.

Pacific. As done in DeMaria and Kaplan (1994b) and Whitney and Hobgood (1997), the translational speed was subtracted from the maximum sustained surface wind of the best track for each storm. The resultant surface wind is defined as the intensity of the TC. The SST at a given time at the TC center is linearly interpolated in time from the weekly Reynolds SST and is interpolated in space using a bicubic spline interpolation.

As seen in Fig. 1a, which shows the scatter diagram of storm intensity versus SST, strong TCs occurred only over high SSTs, as found in other ocean basins (DeMaria and Kaplan 1994b; Whitney and Hobgood 1997). A large number of weak TCs over the high SSTs rep- resent the early stages of TC development in the Tropics. This also explains the fact that most intense TCs are not collocated with the warmest SST because of their poleward movement after formation. A considerable number of TCs occurred over SSTs below $25^{\circ} \mathrm{C}$, indicating that although TCs can only form over SST warmer than $26^{\circ} \mathrm{C}$ (Gray 1968), once they develop, they can survive over the oceans with lower SSTs. These storms, however, were generally weak or were experiencing extratropical transition.

To quantify the relationship between TC intensity and SST, we stratified the observations to each $1^{\circ} \mathrm{C} \mathrm{SST}$ group following DeMaria and Kaplan (1994a). Each 
TABLE 2. Properties of the translational speed groups. Here and hereafter Vmax is defined as the translation-corrected intensity of a TC.

\begin{tabular}{|c|c|c|c|c|c|}
\hline $\begin{array}{l}\text { Translational speed } \\
\text { midpoint }\left(\mathrm{m} \mathrm{s}^{-1}\right)\end{array}$ & $\begin{array}{l}\text { No. of } \\
\text { obs }\end{array}$ & $\begin{array}{l}\text { Avg best-track } \\
\text { intensity }\left(\mathrm{m} \mathrm{s}^{-1}\right)\end{array}$ & $\begin{array}{l}\text { Avg top } 50 \% \text { best-track } \\
\text { intensity }\left(\mathrm{m} \mathrm{s}^{-1}\right)\end{array}$ & $\begin{array}{l}\text { Avg intensity } \\
\operatorname{Vmax}\left(\mathrm{m} \mathrm{s}^{-1}\right)\end{array}$ & $\begin{array}{c}\text { Avg top 50\% } \\
\text { intensity } \operatorname{Vmax}\left(\mathrm{m} \mathrm{s}^{-1}\right)\end{array}$ \\
\hline 1.00 & 1010 & 31.69 & 41.36 & 30.30 & 39.98 \\
\hline 3.00 & 3501 & 34.47 & 45.86 & 31.36 & 42.78 \\
\hline 5.00 & 4127 & 35.99 & 48.27 & 31.01 & 43.29 \\
\hline 7.00 & 2604 & 36.77 & 49.39 & 29.89 & 42.55 \\
\hline 9.00 & 1016 & 33.37 & 44.04 & 24.52 & 35.26 \\
\hline 11.00 & 383 & 32.30 & 41.83 & 21.39 & 30.95 \\
\hline 13.00 & 253 & 31.78 & 40.06 & 18.86 & 27.14 \\
\hline 15.00 & 127 & 30.93 & 37.90 & 15.97 & 23.01 \\
\hline 17.00 & 97 & 29.46 & 35.70 & 12.47 & 18.78 \\
\hline 19.00 & 50 & 29.48 & 34.88 & 10.53 & 15.96 \\
\hline 21.00 & 30 & 33.01 & 38.58 & 11.96 & 17.52 \\
\hline 23.00 & 18 & 34.87 & 40.87 & 11.91 & 17.91 \\
\hline 25.00 & 8 & 31.83 & 34.72 & 6.97 & 10.22 \\
\hline 27.00 & 3 & 37.73 & 42.44 & 10.63 & 14.95 \\
\hline 29.00 & 2 & 30.87 & 30.87 & 1.95 & 2.55 \\
\hline 31.00 & 3 & 33.44 & 34.73 & 3.62 & 3.80 \\
\hline
\end{tabular}

observation was assigned to the nearest midpoint SST. Table 1 shows the properties of all 16 groups of SSTs. There were $92 \%$ observations (i.e., 12135 out of 13 232) that were assigned to SST categories greater than $26^{\circ} \mathrm{C}$, about $10 \%-16 \%$ higher than those found by DeMaria and Kaplan (1994a,b) for the Atlantic systems. The highest average intensity of total systems or top $50 \%$ intense systems occurred in the $27.5^{\circ} \mathrm{C}$ group. Overall, there is a clear trend of increasing average TC intensity with increasing SST until a decline occurs at the very high SST of $27.5^{\circ} \mathrm{C}$, similar to the results for Atlantic TCs found by DeMaria and Kaplan (1994b).

The maximum intensity, the 95th, 90th, and 50th intensity percentiles for each $1^{\circ} \mathrm{C}$ SST group exhibit an exponential function of SST (Fig. 1b), similar to that for Atlantic TCs shown by DeMaria and Kaplan (1994b). Therefore, an empirical MPI as a function of SST similar to that developed by DeMaria and Kaplan (1994b) can be constructed for the western North Pacific TCs. Instead of using the climatological SST in DeMaria and Kaplan (1994b), we used the Reynolds weekly SST linearly interpolated in time and spatially interpolated to the TC center following the best track. The maximum intensity (surface sustained maximum wind speed) can be fitted as an exponential function of SST:

$$
\mathrm{MPI}=A+B e^{C\left(\mathrm{SST}-T_{0}\right)},
$$

where $A=15.69 \mathrm{~m} \mathrm{~s}^{-1}, B=98.03 \mathrm{~m} \mathrm{~s}^{-1}, C=$ $0.1806^{\circ} \mathrm{C}^{-1}$, and $T_{0}=30.0^{\circ} \mathrm{C}$. The fitted curve is given in Fig. 1. This exponential function is the same as that obtained by DeMaria and Kaplan (1994b) for Atlantic TCs. However, the fitted constants $A, B$, and $C$ are different in the two basins (they are $28.2 \mathrm{~m} \mathrm{~s}^{-1}, 55.8$ $\mathrm{m} \mathrm{s}^{-1}$, and $0.1813^{\circ} \mathrm{C}^{-1}$, respectively, for the Atlantic). The slope for the western North Pacific is steeper than that for Atlantic, indicating more intense TCs on the high SST side but weaker TCs on the cold SST side over the western North Pacific (Fig. 1a). Note that this fitted empirical MPI not only includes the thermodynamic effect of SST but also implicitly includes the dynamical effects from environmental flow and vertical shear since the latter are not independent of SST over the region, especially north of $30^{\circ} \mathrm{N}$ (see the discussion in section $6 a$ ).

The outflow temperature does not appear explicitly in the empirical MPI equation (2). Figure 2a shows the scatter diagram of outflow temperature versus SST. Overall the outflow temperature and SST are negatively correlated but with a relatively wide spread. However, the thermodynamic efficiency defined in Eq. (1) is approximately a linear function of SST (Fig. 2b). Therefore, the empirical MPI given in (2) can be considered implicitly including both the effect of the outflow temperature and the dynamical control. A more generalized MPI incorporating the outflow temperature and environmental dynamical control will be developed in section 6 .

\section{Effect of storm translation}

\section{a. Translational speed and TC intensity}

Figure 3a shows the scatter diagram of TC intensity without correction of storm translation against the storm translational speed for the western North Pacific TCs during 1981-2003. We can see an overall trend of 

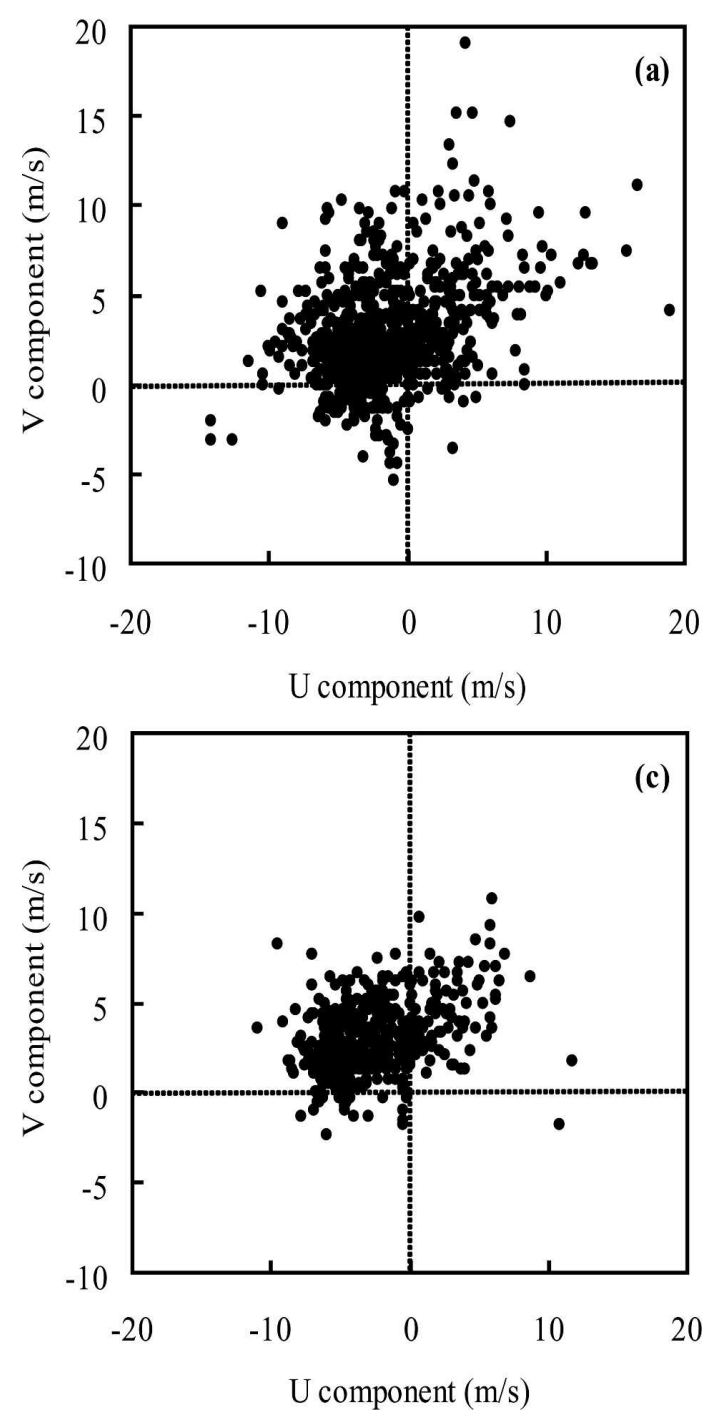

decreasing upper-bound intensity with increasing translational speed, a feature similar to that in Australian region (Wang and $\mathrm{Wu}$ 2004). Very intense TCs (with maximum surface sustained wind speed greater than 65 $\mathrm{m} \mathrm{s}^{-1}$ ) could only develop under a narrow range of translational speeds between 3 and $8 \mathrm{~m} \mathrm{~s}^{-1}$. This implies that either too fast or too slow motion seems to prevent TCs from being too strong. Note that the less dense dots with large translational speeds are mainly related to the storms that recurved into the strong midlatitude westerly and were experiencing extratropical transition. These features remain unchanged even after the translational speed was subtracted from the besttrack intensity (Fig. 3c).

To quantify the general trends seen in Figs. 3a,c, we stratified the observations based on each $2 \mathrm{~m} \mathrm{~s}^{-1}$ translational speed group (Table 2). Similar to the results shown in Table 1 for SST, here each observation was

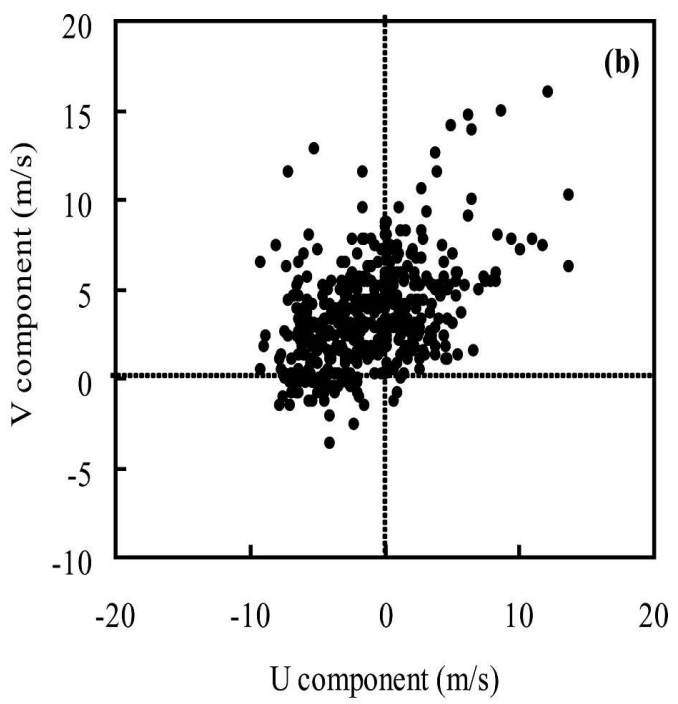

FIG. 4. The $U$ and $V$ components $\left(\mathrm{m} \mathrm{s}^{-1}\right)$ of translation for (a) tropical storms $\left(\mathrm{Cmax}<32 \mathrm{~m} \mathrm{~s}^{-1}\right)$, (b) minor typhoons $\left(32 \mathrm{~m} \mathrm{~s}^{-1} \leq \mathrm{Cmax}<50 \mathrm{~m} \mathrm{~s}^{-1}\right)$, and (c) strong typhoons $\left(\mathrm{Cmax} \geq 50 \mathrm{~m} \mathrm{~s}^{-1}\right)$ over the western North Pacific during 1981-2003, where Cmax is the lifetime peak intensity $\left(\mathrm{m} \mathrm{s}^{-1}\right)$ of individual TCs.

assigned to the nearest midpoint translational speed group. About 93\% observations (i.e., 12258 out of 13 232) were assigned to the groups with translational speed slower than or equal to $10 \mathrm{~m} \mathrm{~s}^{-1}$. The highest average translation-corrected intensity of the total systems or the top $50 \%$ intense systems occurred in the 5 $\mathrm{m} \mathrm{s}^{-1}$ translational speed group. The corresponding maximum TC intensity and the 95th, 90th, and 50th intensity percentiles for each $2 \mathrm{~m} \mathrm{~s}^{-1}$ translational speed group are shown in Figs. 3b,d. Since our focus is mainly on the possible effect of translational speed on the maximum TC intensity, we are mostly interested in the top $90 \%$ intense TCs. Regardless the subtraction of the translational speed or not, the most intense TCs (the top 90\%) generally occur in a narrow range of 3-8 $\mathrm{m} \mathrm{s}^{-1}$ and decreases with both the increase and the decrease of the translational speed (Figs. 3b,d). Note that the results for translational speed large than 20 

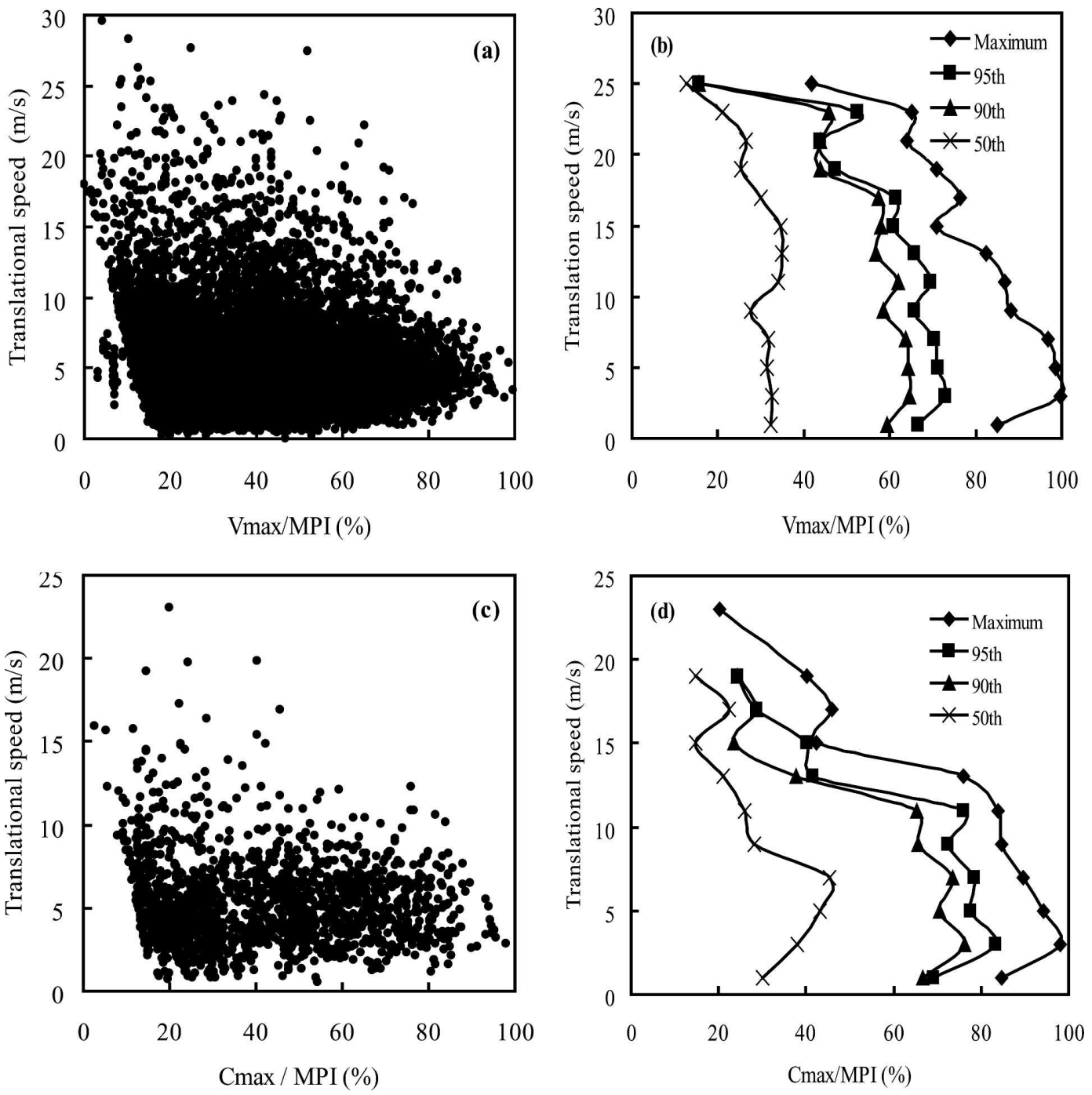

FIG. 5. Scatter diagram of (a) relative intensity (100\% Vmax/MPI) and (c) lifetime relative peak intensity $(100 \%$ Cmax/MPI) against translational speed $\left(\mathrm{m} \mathrm{s}^{-1}\right)$ over the western North Pacific during 1981-2003. (Both Vmax and Cmax in $\mathrm{m} \mathrm{s}^{-1}$ are corrected from storm translation.) (b), (d) The corresponding maximum relative intensity and the 95 th, 90th, and 50th relative intensity percentiles for each $2 \mathrm{~m} \mathrm{~s}^{-1}$ translational speed group.

$\mathrm{m} \mathrm{s}^{-1}$ are not representative since there are too few samples.

The general feature discussed above can be explained based on previous theoretical and numerical studies. If TCs are moving too slow, oceanic cooling induced by turbulent mixing generated by surface wind stress curl under the TC will disrupt the intensification (Schade and Emanuel 1999; Schade 2000), while if they move too fast the resulting asymmetric structure will also inhibit intensification as shown by Peng et al. (1999). In this regard, we can simply assume that the storm translation adds a wavenumber-1 wind asymmetry to the axially symmetric cyclone.

In view of energetic consideration (Emanuel 2000), the contribution by the asymmetric component to the volume-integrated entropy flux, which depends linearly on the absolute value of the ground-relative wind, tends to be zero if the exchange coefficient and boundary layer entropy are quasi symmetric about the storm center. However, the asymmetric component in the ground-relative wind field can have a net contribution to the volume-integrated surface frictional dissipation rate, which varies as the cube of the ground-relative wind. As a result, the net frictional dissipation rate implies a weaker storm having a considerable movement than that implied from the axisymmetric theoretical MPI (Emanuel 1995, 2000).

It is also interesting to examine the storm translation 
when they reached their lifetime peak intensity. Figure 4 shows $U$ and $V$ components of translation for three categories of TCs, namely, tropical storms Cmax $<32$ $\mathrm{m} \mathrm{s}^{-1}$, minor typhoons $\left(32 \mathrm{~m} \mathrm{~s}^{-1} \leq \mathrm{Cmax}<50 \mathrm{~m} \mathrm{~s}^{-1}\right)$, and strong typhoons $\left(\mathrm{Cmax} \geq 50 \mathrm{~m} \mathrm{~s}^{-1}\right.$ ), where Cmax is the lifetime peak intensity after the subtraction of translational speed. For all three categories, a majority of storms reached their lifetime peak intensity in the upper-left quadrant, indicating a northwestward motion of the storms, especially for strong typhoons (Fig. 4c), which also had relatively slow translational speed compared to the tropical storms (Fig. 4a). Evans and McKinley (1998) showed a similar feature in the region and found that about $80 \%$ of the western North Pacific TCs reach their lifetime maximum intensity prior to recurvature. Note that there were still a considerable number of storms that reached their peak intensity after their recurvature with north-northeastward motion for all three categories (Fig. 4).

\section{b. Translational speed and relative intensity}

The dependence of TC intensity on translational speed seen in Fig. 3 also includes the effect of SST since most of the fast movers could be located in the midlatitudes after recurvature over cold SSTs. To partially isolate the effect of translational speed on TC intensity from that of SST, we introduce the so-called relative intensity defined as the percentage of the translationcorrected TC intensity (Vmax) to the SST-determined MPI (100\% Vmax/MPI) and the relative lifetime peak intensity defined as the percentage of the translationcorrected lifetime peak intensity to the SST-determined MPI at the time of peak intensity (100\% Cmax/MPI). Note that the translational speed has also been removed already in the SST-determined MPI in (2).

Figure 5 shows the scatter diagrams of the relative intensity (Fig. 5a) and the lifetime relative peak intensity (Fig. 5c) versus translational speed, together with the corresponding maximum, the 95th, 90th, and 50th relative intensity and relative lifetime peak intensity percentiles for each $2 \mathrm{~m} \mathrm{~s}^{-1}$ translational speed group (Figs. 5b,d). The general properties of the translational speed groups for the relative intensity and lifetime relative peak intensity are also listed in Table 2. In general, there is a trend of increasing relative TC intensity with decreasing translational speed except for high relative intensity at low translational speeds (Figs. 5a,c), similar to the results shown in Figs. 3a,c, indicating an overall negative effect of translational speed on TC intensity. Dots with low relative intensity and low translational speed in Fig. 5a represent the early development stage of TCs that are over high SSTs and thus have large MPI.

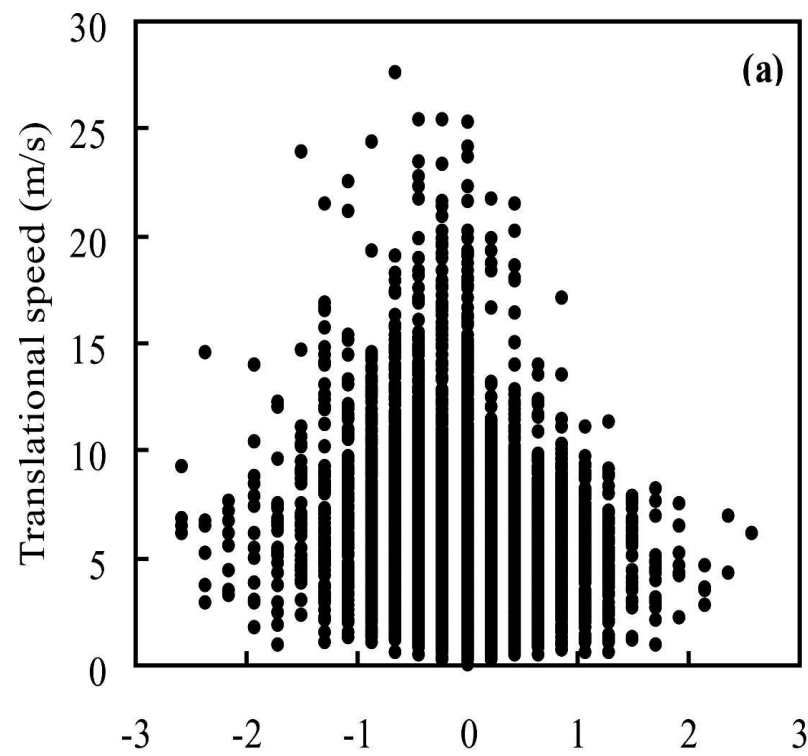

Rate in intensity change (A positive number indicates TC intensity is increasing)

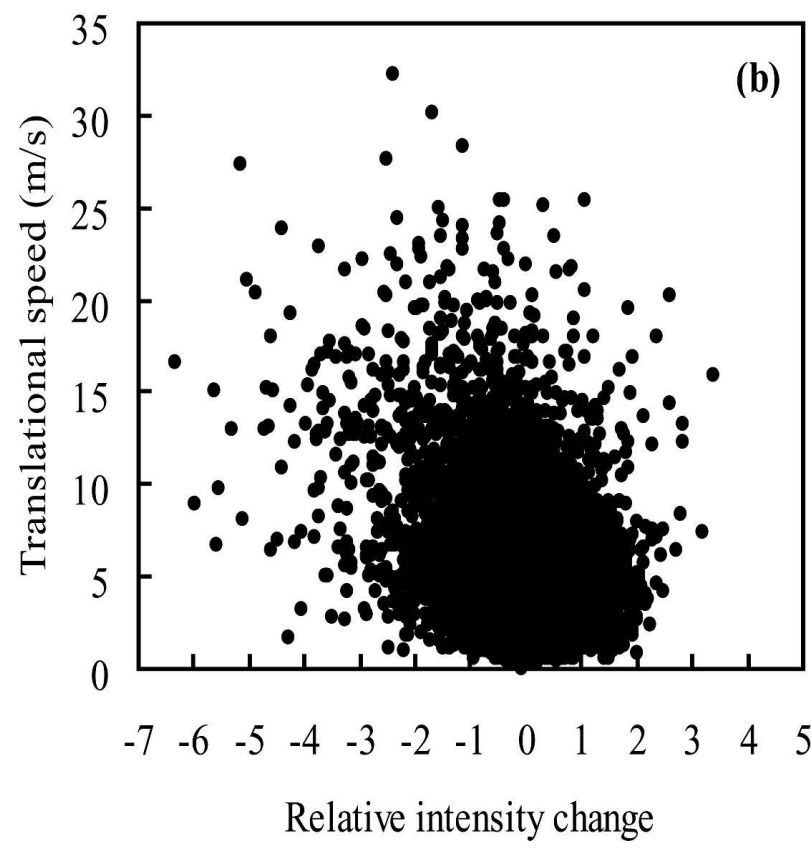

FIG. 6. Scatter diagrams of (a) rate in intensity change $\left(\mathrm{m} \mathrm{s}^{-1} \mathrm{~h}^{-1}\right)$ and (b) rate in relative intensity change $\left(\% \mathrm{~h}^{-1}\right)$ against storm translational speed $\left(\mathrm{m} \mathrm{s}^{-1}\right)$, respectively, for TCs over the western North Pacific during 1981-2003.

The relationship between the lifetime relative peak intensity and translational speed (Figs. 5c,d) displays a similar feature to the relative intensity seen in Figs. 5a,b. Now the lifetime relative peak intensity occurs at relatively low translational speeds, consistent with the fact that most TCs reach their lifetime peak intensity 

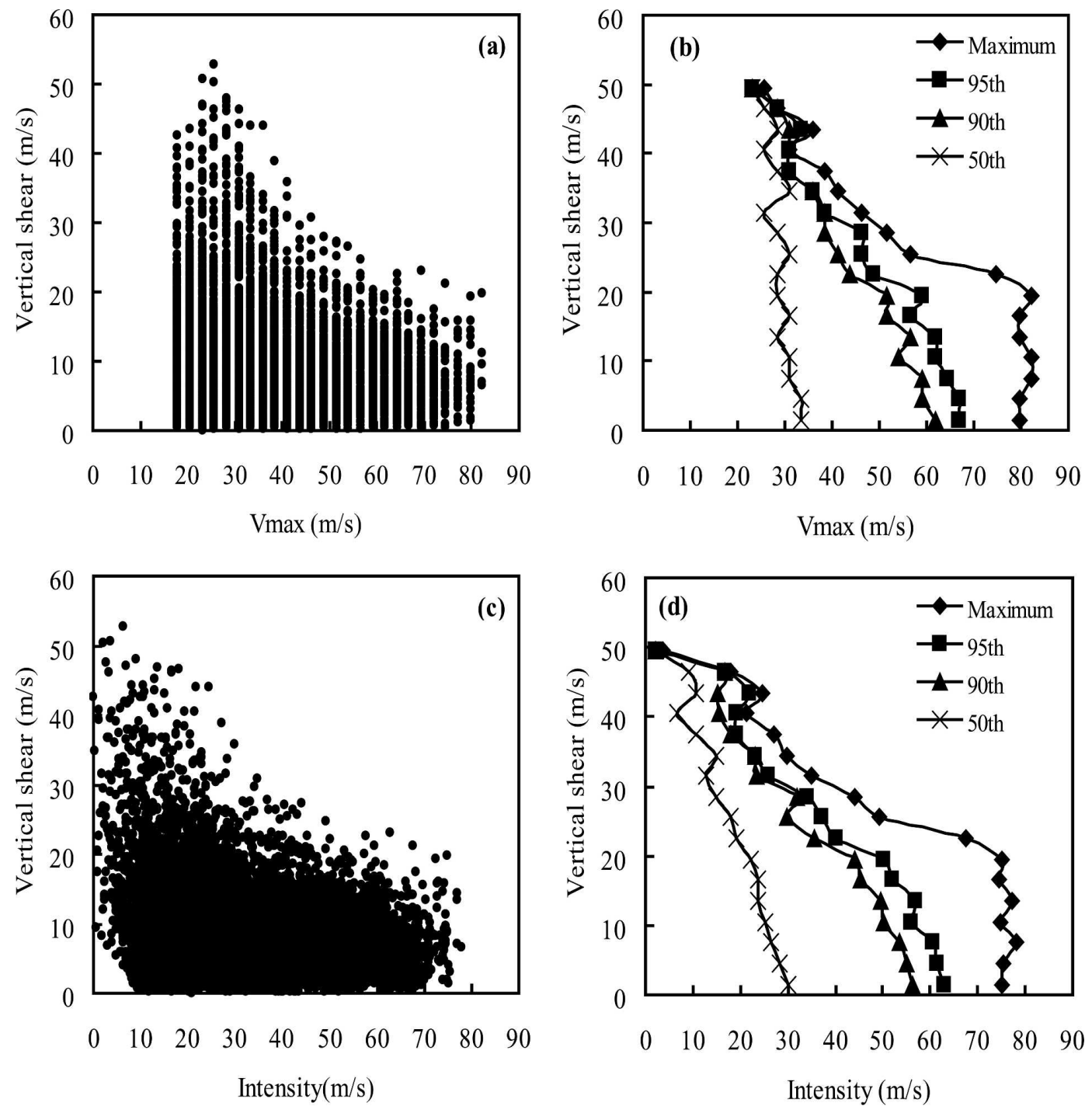

FIG. 7. Scatter diagrams of TC intensity $\left(\mathrm{m} \mathrm{s}^{-1}\right)$ vs vertical shear $\left(\mathrm{m} \mathrm{s}^{-1}\right)$ from (a) the best-track intensity and (c) that corrected by subtracting storm translational speed for TCs over the western North Pacific during 1981-2003, and (b), (d) the corresponding maximum intensity and the 95th, 90th, and 50th intensity percentiles for each $2 \mathrm{~m} \mathrm{~s}^{-1}$ vertical shear group.

just prior to recurvature where the environmental steering flow is generally weak (Evans and McKinley 1998). Although the samples are too small to be used accurately to assess the translational speed on the lifetime peak TC intensity (Fig. 5c), a consistent trend is still clear. As seen from Fig. 5d, the maximum intensity, the 95th and 90th percentiles of the lifetime relative peak intensity for each $2 \mathrm{~m} \mathrm{~s}^{-1}$ translational speed group, all tend to increase with the decrease in translational speed, indicating that the storm translation is one of the limiting factors to their intensity. Note that a lot of storms can only reach a small portion of their MPI even at low translational speeds (Figs. 5a,c), im- plying other factors prohibiting the TC from intensifying.

\section{c. Translational speed and intensification rate}

Since the translational speed affects the TC intensity, a natural question arises as to whether any relationship exists between the translational speed and intensification rate. To address this issue, we examined the rate of intensity change versus the translational speed and show the scatter diagram in Fig. 6a. Generally the intensification rate (positive) increases with decreasing translational speed, indicating an overall negative effect of fast translation on TC intensification. Note that both 
TABLE 3. Properties of the vertical shear groups.

\begin{tabular}{|c|c|c|c|c|c|}
\hline $\begin{array}{l}\text { Vertical shear } \\
\text { midpoint }\left(\mathrm{m} \mathrm{s}^{-1}\right)\end{array}$ & $\begin{array}{l}\text { No. of } \\
\text { obs }\end{array}$ & $\begin{array}{l}\text { Avg best-track } \\
\text { intensity }\left(\mathrm{m} \mathrm{s}^{-1}\right)\end{array}$ & $\begin{array}{l}\text { Avg top } 50 \% \text { best-track } \\
\text { intensity }\left(\mathrm{m} \mathrm{s}^{-1}\right)\end{array}$ & $\underset{\left(\mathrm{m} \mathrm{s}^{-1}\right)}{\operatorname{Avg} \operatorname{Vmax}}$ & $\begin{array}{c}\text { Avg top } 50 \% \text { Vmax } \\
\left(\mathrm{m} \mathrm{s}^{-1}\right)\end{array}$ \\
\hline 1.00 & 707 & 38.10 & 50.88 & 33.09 & 45.97 \\
\hline 3.00 & 1658 & 37.11 & 49.67 & 32.29 & 45.02 \\
\hline 5.00 & 2286 & 36.07 & 48.31 & 31.11 & 43.44 \\
\hline 7.00 & 2293 & 35.49 & 47.54 & 30.42 & 42.51 \\
\hline 9.00 & 2007 & 34.19 & 45.45 & 29.12 & 40.55 \\
\hline 11.00 & 1560 & 33.47 & 44.39 & 28.25 & 39.24 \\
\hline 13.00 & 1023 & 33.26 & 44.07 & 27.49 & 38.44 \\
\hline 15.00 & 612 & 33.80 & 44.36 & 27.47 & 38.40 \\
\hline 17.00 & 302 & 31.49 & 40.03 & 24.28 & 33.18 \\
\hline 19.00 & 233 & 32.52 & 42.78 & 24.51 & 34.71 \\
\hline 21.00 & 154 & 32.54 & 41.96 & 22.87 & 32.29 \\
\hline 23.00 & 104 & 30.62 & 38.83 & 20.30 & 29.27 \\
\hline 25.00 & 74 & 30.72 & 37.15 & 18.98 & 25.81 \\
\hline 27.00 & 54 & 31.39 & 39.25 & 20.08 & 28.74 \\
\hline 29.00 & 33 & 27.20 & 32.38 & 13.34 & 18.55 \\
\hline 31.00 & 29 & 29.62 & 34.98 & 14.80 & 21.56 \\
\hline 33.00 & 29 & 29.27 & 32.92 & 14.89 & 19.26 \\
\hline 35.00 & 13 & 27.30 & 32.70 & 14.42 & 19.85 \\
\hline 37.00 & 23 & 26.28 & 29.58 & 10.56 & 14.10 \\
\hline 39.00 & 7 & 27.93 & 31.51 & 11.97 & 18.45 \\
\hline 41.00 & 10 & 25.46 & 28.81 & 10.66 & 16.34 \\
\hline 43.00 & 10 & 27.01 & 30.87 & 12.22 & 17.06 \\
\hline 45.00 & 3 & 26.58 & 28.29 & 10.57 & 11.82 \\
\hline
\end{tabular}

rapid intensification and weakening are rare events and thus do not represent the majority. However, a robust feature in Fig. 6a is the fact that few TCs intensified when they moved with translational speeds of more than $15 \mathrm{~m} \mathrm{~s}^{-1}$, especially for storms with maximum sustained surface winds larger than $32 \mathrm{~m} \mathrm{~s}^{-1}$ (not shown).

Note that most TCs intensified before recurvature in the tropical and subtropical oceans where the environmental steering flow is relatively weak compared to the strong westerlies in the midlatitudes after recurvature. Therefore, the trend seen in Fig. 6a could be partially attributed to the effect of cold SST for fast movers after their recurvature. To possibly isolate the effect of storm translation from the effect of SST, we examined the rate of relative intensity (100\% Vmax/MPI) change against the translational speed (Fig. 6b). Consistent with the results shown in Fig. 6a, the intensifying storms have relatively low translational speed while the majority of the weakening storms have relatively high translational speeds. Therefore, overall the fast translation prohibits TC intensification.

\section{Effect of vertical shear}

\section{a. Vertical shear and TC intensity}

Figure 7 gives the scatter diagrams of TC intensity without (Fig. 7a) and with (Fig. 7c) translation correc- tion against the vertical shear and the corresponding maximum intensity, and the 95th, 90th, and 50th intensity percentiles for each $2 \mathrm{~m} \mathrm{~s}^{-1}$ vertical shear group (Figs. 7b,d). The stratification and the general properties of the vertical shear groups for both the best-track intensity and the translation-corrected intensity are listed in Table 3. In both cases, there is a general trend of increasing TC intensity with decreasing vertical shear. Very strong TCs can survive in quite strong vertical shears but very few intense TCs occur when the vertical shear is larger than $20 \mathrm{~m} \mathrm{~s}^{-1}$. Zehr (1992) found that TCs could not form if the vertical shear was larger than $12.5 \mathrm{~m} \mathrm{~s}^{-1}$ over the western North Pacific. Our results show that once TCs are strong enough they could resist quite strong vertical shear effect, in support of the results of Wang et al. (2004), who found that well-developed TCs could resist unidirectional vertical shear as large as $15-18 \mathrm{~m} \mathrm{~s}^{-1}$. Note that the 95th and 90th intensity percentiles for each $2 \mathrm{~m} \mathrm{~s}^{-1}$ vertical shear groups (Figs. 7b,d) show a nearly linear decrease with increasing vertical shear, indicating an overall negative effect of vertical shear on TC intensity.

Figure 8 shows the scatter diagram of $U$ and $V$ shear components for strong and weak TCs south and north of $30^{\circ} \mathrm{N}$, respectively. We can see that large vertical shear north of $30^{\circ} \mathrm{N}$ mostly occurs in the upper-right quadrant (Figs. 8c,d), indicating dominant southwesterly vertical shear ahead of midlatitude westerly troughs 

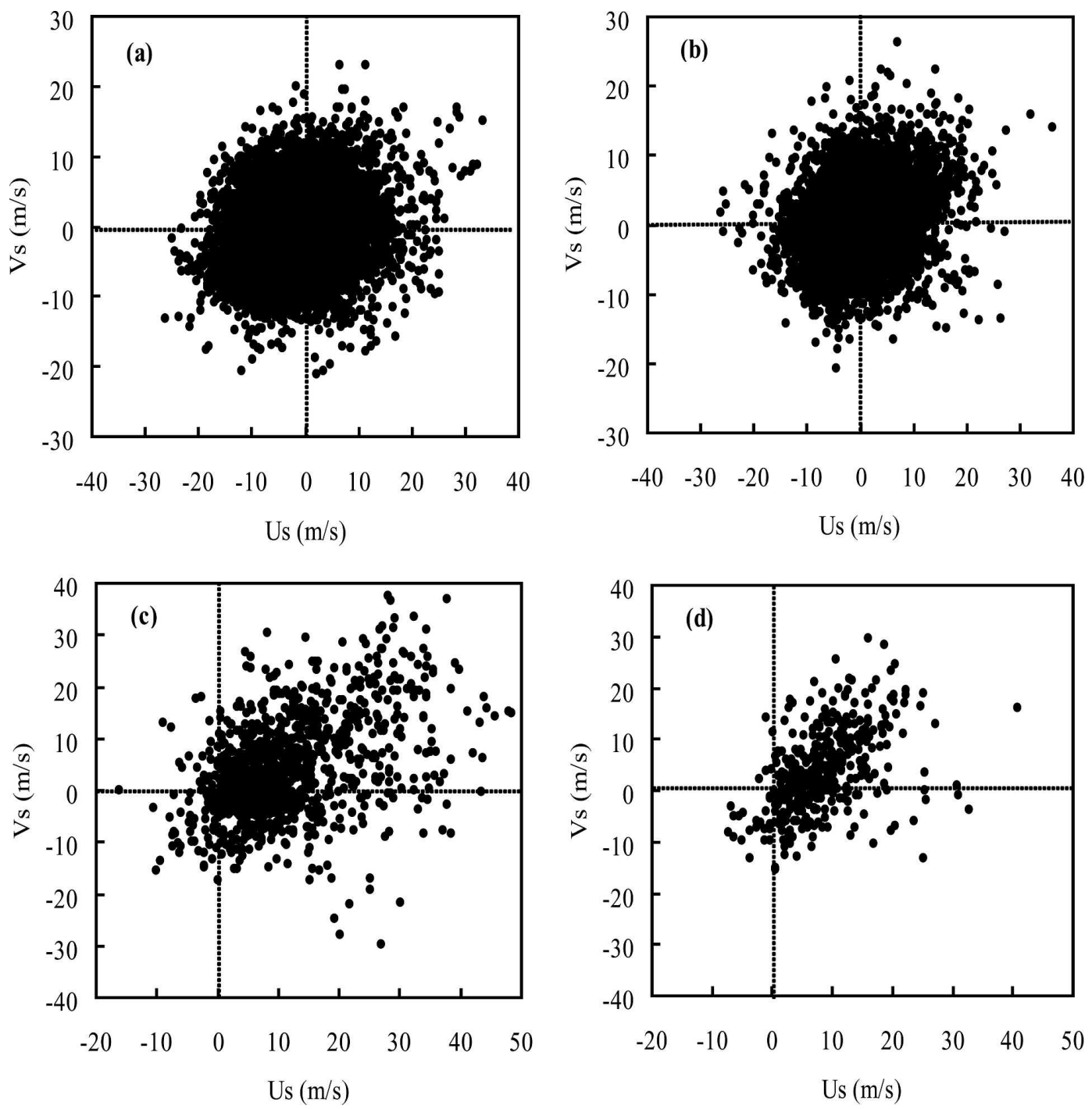

FIG. 8. Scatter diagrams of vertical shear components (Us vs Vs) for (a), (c) tropical storms (Vmax $<32 \mathrm{~m} \mathrm{~s}^{-1}$ ) and (b), (d) typhoons (Vmax $\geq 32 \mathrm{~m} \mathrm{~s}^{-1}$ ) (a), (b) south and (c), (d) north of $30^{\circ} \mathrm{N}$ over the western North Pacific during 1981-2003.

in the mid- to upper troposphere over East Asia. However, south of $30^{\circ} \mathrm{N}$, there is no dominant directional shear (Figs. 8a,b). Compared with the weak storms (Figs. 8a,c), the strong storms correspond to weaker vertical shear (Figs. 8b,d), indicative of an overall negative effect of vertical shear on TC intensity.

\section{b. Vertical shear and relative intensity}

As in the case for the translational speed discussed in section $4 \mathrm{a}$, the dependence of TC intensity on the vertical shear also involves the effect of SST because large vertical shear mostly occur in the midlatitudes where SSTs are generally low. To partially isolate the vertical shear effect from the effect of SST, we examined the relationship between the vertical shear and the relative intensity (100\% Vmax/MPI) and lifetime relative peak intensity (100\% Cmax/MPI), respectively. The scatter diagrams in Figs. 9a,c show a general increasing trend of the upper bounds of both the relative intensity and lifetime relative peak intensity as the vertical shear decreases. The maximum, 95th, and 90th relative intensity and lifetime relative intensity percentiles for each 2 $\mathrm{m} \mathrm{s}^{-1}$ vertical shear group (Figs. 9b,d) all decrease predominantly with increasing vertical shear, indicative of an overall negative effect of vertical shear on TC intensity. However, the rate of change (slope) is slower than that seen in Figs. 7b,d, indicating the possible contribution by SST in Fig. 7 because the large vertical shears are usually correlated with cold SSTs in the midlatitudes. Note that except for a few cases most storms 

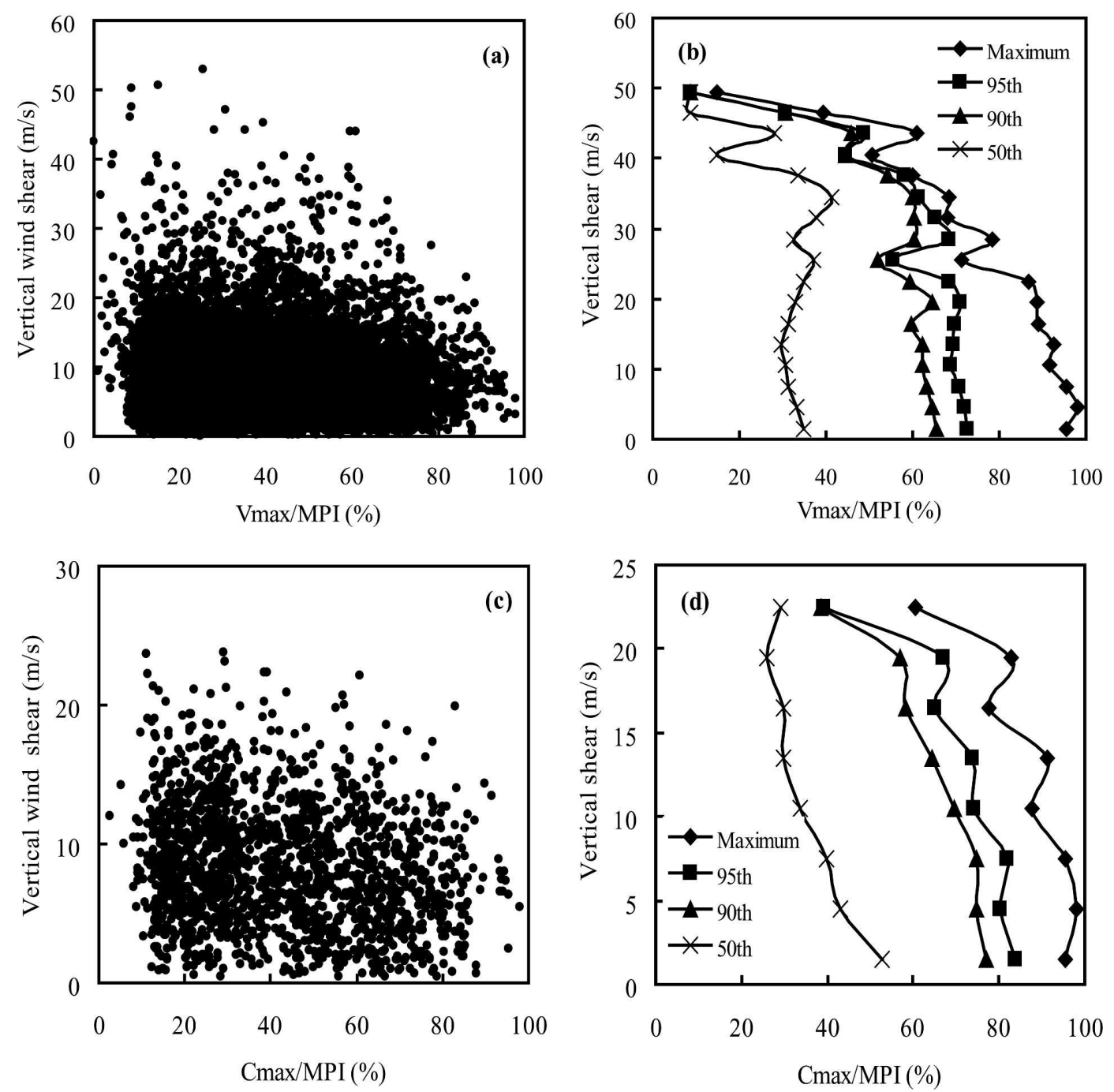

FIG. 9. Scatter diagrams of (a) relative intensity (100\% Vmax/MPI) and (c) lifetime relative peak intensity (100\% Cmax/MPI) against vertical wind shear $\left(\mathrm{m} \mathrm{s}^{-1}\right)$, respectively, for TCs over the western North Pacific during 1981-2003 and (b), (d) the corresponding maximum relative intensity and the 95th, 90th, and 50th relative intensity percentiles for each $2 \mathrm{~m} \mathrm{~s}^{-1}$ vertical shear group.

reach their lifetime relative peak intensity when the vertical shear is less than $20 \mathrm{~m} \mathrm{~s}^{-1}$ (Fig. 9c). There are still a large number of storms having weak lifetime relative peak intensities under quite weak vertical shear conditions, indicating that vertical shear is not the only prohibiting factor to TC intensity.

\section{c. Vertical shear and intensification rate}

It has been shown in earlier observational studies that the vertical shear has a negative effect on TC intensification (e.g., Gray 1968; Merrill 1988). Figure 10 shows the scatter diagram of the 24-h lagged intensity change against the vertical shear. Our initial assessment showed that the vertical shear usually has a delayed negative effect on TC intensity (the results are similar for 12- and 36-h lags, not shown). We can see that few intensification cases occur when the vertical shear is larger than $20 \mathrm{~m} \mathrm{~s}^{-1}$, consistent with the results discussed in section $6 \mathrm{~b}$ that most TCs reach their lifetime peak intensity with vertical shear less than $20 \mathrm{~m} \mathrm{~s}^{-1}$ (Fig. 9b). Although Zehr (1992) determined a threshold vertical shear of $12.5 \mathrm{~m} \mathrm{~s}^{-1}$ above which no TCs could form, the results in Fig. 10 demonstrate that a large portion of TCs can intensify even with vertical shear larger than $12.5 \mathrm{~m} \mathrm{~s}^{-1}$. In general, however, the upper bound of intensification rate decreases with increasing vertical shear, especially for storms with intensity far from their MPI and having potential to intensify (not shown). Note that the rapid weakening storms are not necessarily related to the very strong vertical shear 


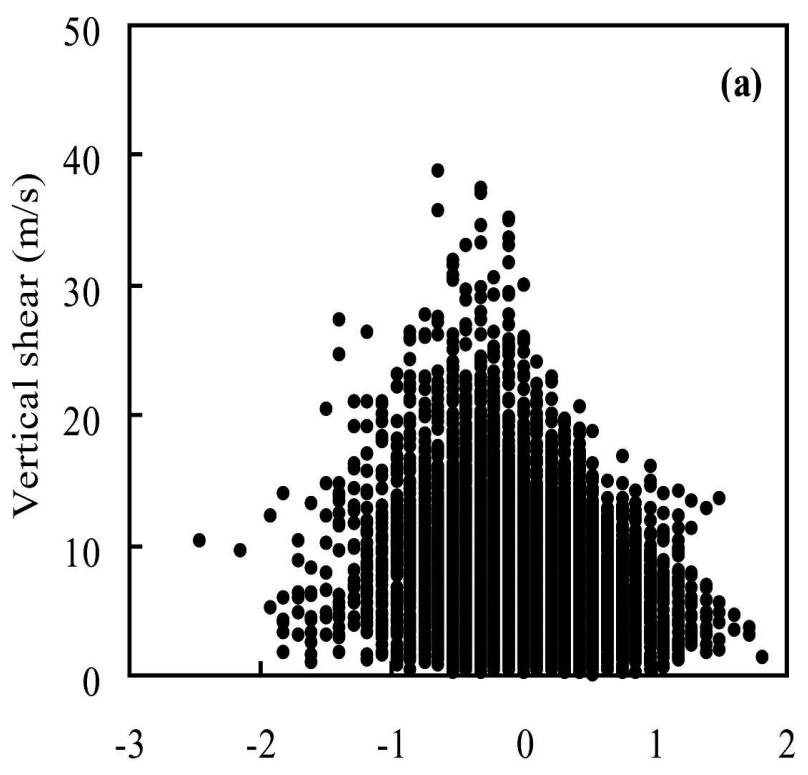

Rate in intensity change (A positive number indicates TC intensity is increasing)

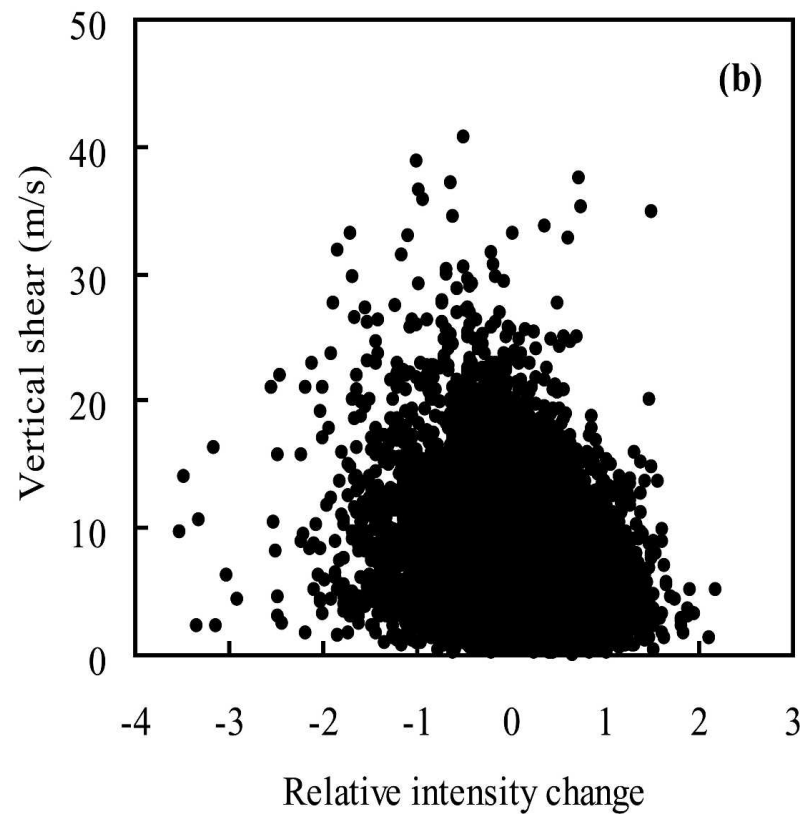

FIG. 10. Scatter diagrams of (a) rate in intensity change $\left(\mathrm{m} \mathrm{s}^{-1} \mathrm{~h}^{-1}\right)$ and (b) rate in relative intensity change $\left(\% \mathrm{~h}^{-1}\right)$ against vertical wind shear $\left(\mathrm{m} \mathrm{s}^{-1}\right)$, respectively, for TCs over the western North Pacific during 1981-2003. Note that both intensity and intensity change are 24-h lagged relative to the vertical shear.

(Fig. 10a), indicating that factors other than the vertical shear are also responsible for the rapid weakening of TCs.

The results shown in Fig. 10a could be partially attributed to the cold SST effect. To possibly isolate the
SST effect, we examined the relationship between vertical shear and the relative intensity (100\% Vmax/MPI) change (Fig. 10b). Consistent with the results shown in Fig. 10a, the intensification rate increases with decreasing vertical shear while the majority of the weakening storms experience relatively strong vertical shears. Therefore, overall strong vertical shear prohibits rapid intensification and most likely results in TC weakening, which is consistent with previous studies.

\section{Dynamical control of TC MPI}

\section{a. The combined effect of translation and vertical shear}

In sections 4 and 5, we have discussed the effects of translation and vertical shear, separately, on TC intensity. Since both the vertical wind shear and translational speed have negative effects on TC intensity, their combined effect can be evaluated by TC intensity or relative intensity (100\% Vmax/MPI) and TC lifetime peak intensity or lifetime peak relative intensity $(100 \%$ Cmax/MPI) against certain measure of the magnitude of vertical shear and translation. After intensive subjective tests, we find that the following definition is a good candidate:

$$
U_{\mathrm{ST}}=\sqrt{0.6 V_{\text {shear }}^{2}+\left(V_{\text {trans }}-5\right)^{2}},
$$

where $V_{\text {shear }}$ and $V_{\text {trans }}$ are vertical shear and translational speed, respectively. Note that a constant $5 \mathrm{~m} \mathrm{~s}^{-1}$ is subtracted from the translational speed to take into account the negative effect of ocean mixing for slowmoving TCs (see Figs. 3 and 5). The constant 0.6 for the square of vertical shear reflects the slightly smaller slope of the top intensity percentiles for each vertical shear group shown in Figs. 7 and 9 than that for translational speed shown in Figs. 3 and 5.

Figure 11 gives the scatter diagrams of TC intensity (Fig. 11a) and lifetime peak intensity (Fig. 11c) against $U_{\mathrm{ST}}$ defined in (3), and the corresponding maximum intensity, and the 95th, 90th, and 50th intensity percentiles for each $3 \mathrm{~m} \mathrm{~s}^{-1} U_{\mathrm{ST}}$ group (Figs. 11b,d), respectively. The stratification and the general properties of the $U_{\mathrm{ST}}$ groups for TC intensity (Vmax) and lifetime peak intensity (Cmax) are given in Tables 4 and 5, respectively. We see that both the average (lifetime peak) intensity and the average top 50\% (lifetime peak) intensity, and the top intensity percentiles of both the intensity and lifetime peak intensity for each $U_{\mathrm{ST}}$ group increase as the combined magnitude $U_{\mathrm{ST}}$ of vertical shear and translation decreases, consistent with the individual effects from translational speed (Fig. 3) and vertical shear (Fig. 7), respectively. Similar features can 

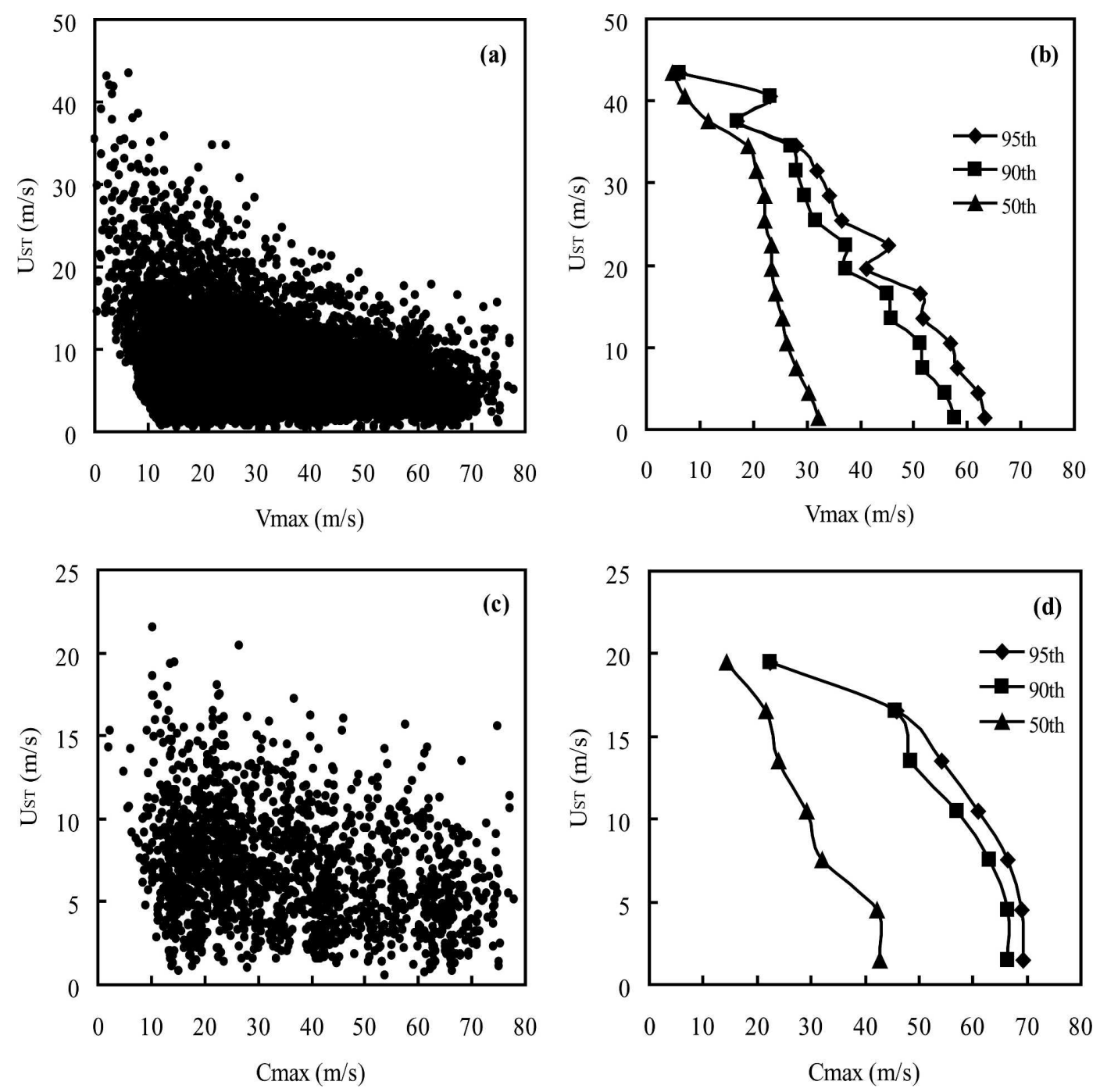

FIG. 11. Scatter diagrams of (a) intensity (Vmax) and (c) lifetime peak intensity (Cmax) against the combined effect of storm translation and environmental vertical shear $\left(\mathrm{m} \mathrm{s}^{-1}\right)$ over the western North Pacific during 19812003, together with (b), (d) the corresponding 95th, 90th, and 50th intensity percentiles for each $3 \mathrm{~m} \mathrm{~s}^{-1}$ combined shear and translational speed group.

be found for the relative intensity (100\% Vmax/MPI) and the lifetime relative peak intensity $(100 \% \mathrm{Cmax} /$ MPI) as shown in Fig. 12 and listed in Tables 4 and 5. These results further demonstrate that it is feasible to add one more dimension to the upper limit of possible TC MPI by introducing the combined negative effect of vertical shear and translational speed.

\section{b. A new empirical MPI incorporating environmental dynamical control}

Based on the above results, we can construct a new empirical MPI incorporating the environmental dynamical control, namely, the combined effect of vertical shear and translation. Since the dynamical control is negative to the TC maximum intensity, we can introduce an attenuation factor $\eta$ defined as

$$
\eta=1 /\left(1+\sin \varphi U_{\mathrm{ST}} / U_{0}\right),
$$

where $U_{0}$ is taken to be $45 \mathrm{~m} \mathrm{~s}^{-1}$ and used to normalize the combined measure of the magnitude of vertical shear and translation. Parameter $\eta$ represents a nondimensional attenuation factor due to the combined negative effect of translation and vertical shear. Note that a latitude factor, $\sin \varphi$, is introduced to take into account the possible effect of large-scale atmospheric conditions, such as the lowering of tropopause and the increasing inertial stability to resist the radial inflow with latitude. As a result, storms moving away from the 
TABLE 4. Properties of the $U_{\mathrm{ST}}$ groups for translation-corrected intensity and related intensity.

\begin{tabular}{cccccc}
\hline $\begin{array}{c}U_{\text {ST }} \text { midpoint } \\
\left(\mathrm{m} \mathrm{s}^{-1}\right)\end{array}$ & $\begin{array}{c}\text { No. of } \\
\text { obs }\end{array}$ & $\begin{array}{c}\text { Avg } \\
\text { Vmax }\left(\mathrm{m} \mathrm{s}^{-1}\right)\end{array}$ & $\begin{array}{c}\text { Avg top 50\% } \\
\text { Vmax }\left(\mathrm{m} \mathrm{s}^{-1}\right)\end{array}$ & $\begin{array}{c}\text { Avg } \\
\text { Vmax/MPI (\%) }\end{array}$ & $\begin{array}{c}\text { Avg top 50\% } \\
\text { Vmax/MPI }(\%)\end{array}$ \\
\hline 1.50 & 1407 & 32.79 & 45.47 & 27.93 & 45.33 \\
4.50 & 4535 & 31.25 & 43.60 & 27.23 & 44.05 \\
7.50 & 3768 & 28.94 & 40.16 & 22.01 & 39.76 \\
10.50 & 1887 & 27.91 & 38.85 & 18.83 & 30.33 \\
13.50 & 925 & 26.35 & 35.93 & 20.00 & 32.14 \\
16.50 & 325 & 25.63 & 35.43 & 19.10 & 30.61 \\
19.50 & 159 & 22.75 & 30.61 & 18.06 & 29.46 \\
22.50 & 86 & 22.44 & 30.53 & 18.59 & 30.48 \\
25.50 & 62 & 20.22 & 27.13 & 11.17 & 17.89 \\
28.50 & 37 & 18.63 & 26.04 & 10.81 & 23.31 \\
31.50 & 16 & 19.38 & 27.14 & 10.51 & 18.18 \\
34.50 & 12 & 16.83 & 25.93 & 12.16 & 15.01 \\
37.50 & 6 & 10.36 & 15.35 & 12.99 & 15.17 \\
40.50 & 4 & 3.18 & 4.57 & 15.15 \\
43.50 & 3 & 3.81 & & & \\
\hline
\end{tabular}

Tropics would be subject to larger negative effect from either translation, or vertical shear, or both.

We define an effective SST ( $\mathrm{SST}_{\text {eff }}$ ) as

$$
\mathrm{SST}_{\text {eff }}=\eta \mathrm{SST} \text {. }
$$

It reads that the positive role of SST in determining the TC MPI is attenuated by the combined negative effect of translational speed and vertical shear. In addition, to maximize the use of information and to extend the applicability of the new empirical MPI, we incorporate the thermodynamic efficiency defined in (1). Figure 13a shows the scatter diagram of the TC intensity divided by the thermodynamic efficiency against the effective SST $\left(\mathrm{SST}_{\text {eff }}\right)$ from observations. Based on Fig. 13a, an exponential function similar to SST-determined MPI given in Eq. (2) can be obtained by fitting the observed maximum TC intensity and the effective SST and the thermodynamic efficiency (see curve in Fig. 13a):

$$
\mathrm{MPI}_{\text {new }}=\varepsilon\left[A^{\prime}+B^{\prime} e^{C^{\prime}\left(\mathrm{SST}_{\mathrm{eff}}-T_{0}\right)}\right] \text {, }
$$

where $A^{\prime}=-63.85 \mathrm{~m} \mathrm{~s}^{-1}, B^{\prime}=206.35 \mathrm{~m} \mathrm{~s}^{-1}, C^{\prime}=$ $0.0516^{\circ} \mathrm{C}^{-1}$, and $T_{0}=30.0^{\circ} \mathrm{C}$. The thermodynamic ef- ficiency is included to make the new MPI applicable to more general thermodynamic conditions, such as other ocean basins or future climate (Henderson-Sellers et al. 1998).

To identify the direct effect of dynamical control on TC MPI, we accordingly modify the original SSTdetermined MPI given in (2) to include the effect of thermodynamic efficiency:

$$
\mathrm{MPI}^{M}=\varepsilon\left[A^{\prime \prime}+B^{\prime \prime} e^{C^{\prime \prime}\left(\mathrm{SST}_{\mathrm{eff}}-T_{0}\right)}\right],
$$

where $A^{\prime \prime}=0.87 \mathrm{~m} \mathrm{~s}^{-1}, B^{\prime \prime}=140.35 \mathrm{~m} \mathrm{~s}^{-1}, C^{\prime \prime}=$ $0.1206^{\circ} \mathrm{C}^{-1}$, and $T_{0}=30.0^{\circ} \mathrm{C}$. Note that we use the superscript " $M$ " to represent the MPI with the modification to include the effect of thermodynamic efficiency (or the outflow temperature). Although (7) represents the empirical MPI as the same as the SST-determined one given in (2), the modified MPI in (7) includes the effect of thermodynamic efficiency explicitly and thus can be used to measure the dynamical control on the TC MPI if it is compared with the new MPI given in (6). Note that (7) includes the thermodynamic control explicitly and the dynamical control implicitly, while the

TABLE 5. Properties of the $U_{\mathrm{ST}}$ groups for translation-corrected lifetime peak intensity and relative peak intensity.

\begin{tabular}{cccccc}
\hline $\begin{array}{c}U_{\mathrm{ST}} \text { midpoint } \\
\left(\mathrm{m} \mathrm{s}^{-1}\right)\end{array}$ & $\begin{array}{c}\text { No. of } \\
\text { obs }\end{array}$ & $\begin{array}{c}\text { Avg } \\
\text { Cmax }\left(\mathrm{m} \mathrm{s}^{-1}\right)\end{array}$ & $\begin{array}{c}\text { Avg top 50\% } \\
\left.\text { Cmax (m s })^{-1}\right)\end{array}$ & $\begin{array}{c}\text { Avg } \\
\text { Cmax/MPI (\%) }\end{array}$ & $\begin{array}{c}\text { Avg top 50\% } \\
\text { Cmax/MPI (\%) }\end{array}$ \\
\hline 1.50 & 285 & 42.16 & 58.09 & 41.28 & 63.59 \\
4.50 & 717 & 40.31 & 56.27 & 37.51 & 58.84 \\
7.50 & 685 & 33.13 & 46.97 & 32.51 & 49.98 \\
10.50 & 400 & 31.44 & 44.37 & 30.70 & 37.35 \\
13.50 & 204 & 27.54 & 37.88 & 23.73 & 41.07 \\
16.50 & 30 & 23.93 & 35.08 & 26.81 & 29.31 \\
19.50 & 5 & 17.41 & 21.05 & 20.43 & 20.14 \\
22.50 & 1 & 10.39 & 10.39 & 20.14 & \\
\hline
\end{tabular}



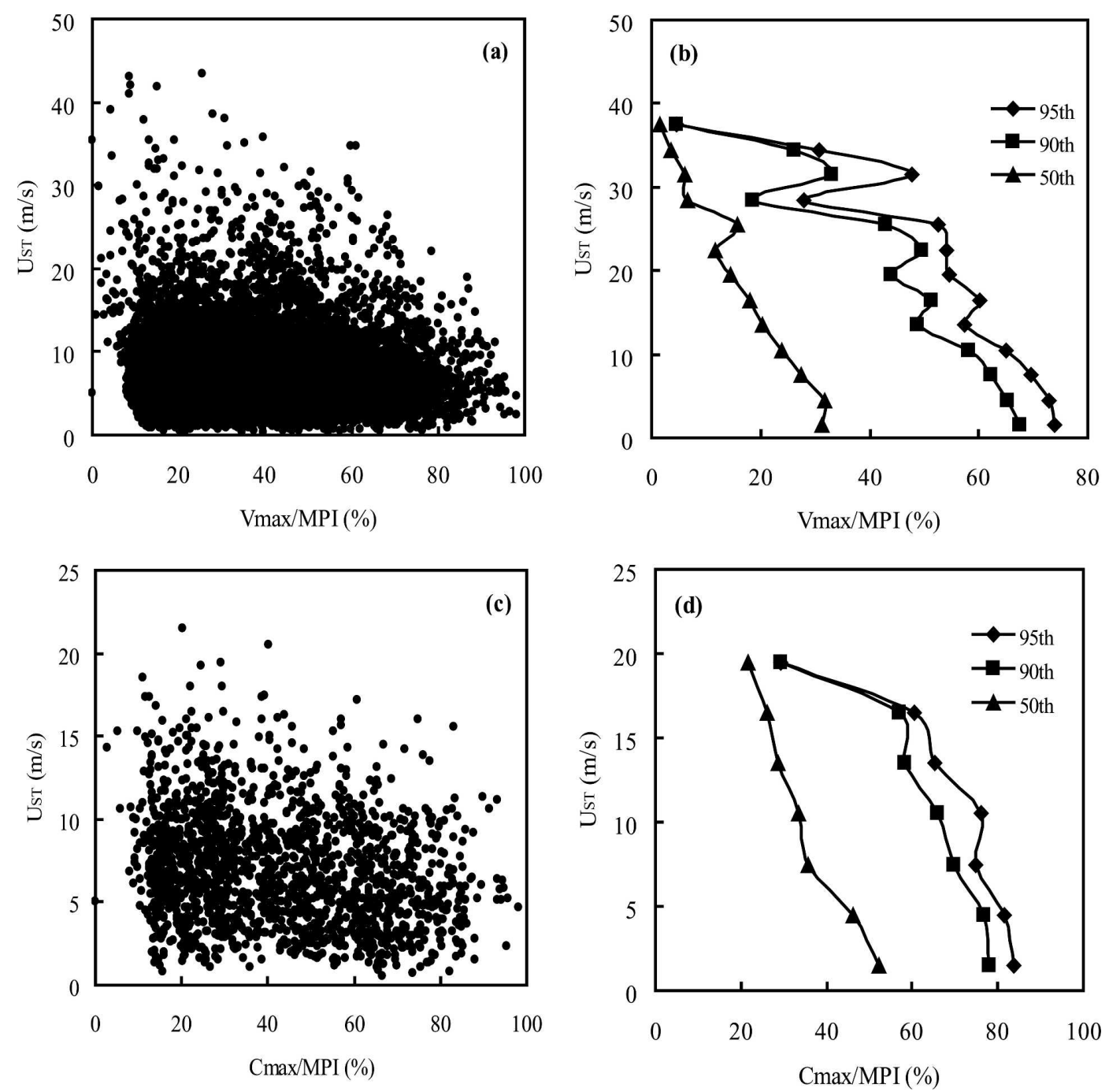

FIG. 12. Scatter diagrams of (a) relative intensity (100\% Vmax/MPI) and (c) relative lifetime peak intensity $\left(100 \%\right.$ Cmax/MPI) against the combined effect of storm translation and environmental vertical shear $\left(\mathrm{m} \mathrm{s}^{-1}\right)$ over the western North Pacific during 1981-2003, together with (b), (d) the corresponding 95th, 90th, and 50th relative intensity percentiles for each $3 \mathrm{~m} \mathrm{~s}^{-1}$ combined shear and translational speed group.

new MPI in (6) explicitly includes both the thermodynamic and dynamical controls of TC maximum intensity.

If we set $\eta=1$ for the new MPI in (6), the difference between the modified MPI in (7) and the new MPI in (6) can be regarded as the limiting effect due to the dynamical control from both the translation and vertical shear. As seen from Fig. 13b, without the environmental dynamical effect $(\eta=1)$, the new MPI, which is purely determined by the thermodynamic conditions of the atmosphere and the ocean surface, is always larger than the MPI defined in (7), which implicitly includes the dynamical effect already. This indicates that the empirical MPI in (7) or (2) underestimates the thermodynamic control of the TC MPI.
The new empirical MPI has several advantages. First it provides a measure of the dynamical control on TC MPI (Fig. 13b). It also gives more accurate MPI estimation since it includes extra information of the limiting factors due to translation and vertical shear. The latter is true since the percentage of TCs reaching the new MPI is higher than that reaching the MPIs without explicitly including the dynamical control (Fig. 14 and Table 6), indicating that the SST-determined MPI overestimates the maximum intensity for some TCs. In addition, as mentioned in section 3, because the thermodynamic efficiency is closely related to SST (Fig. 2b), the inclusion of the thermodynamic efficiency in the modified MPI in (7) only improves the estimation of TC maximum intensity marginally (Fig. 14). As seen 

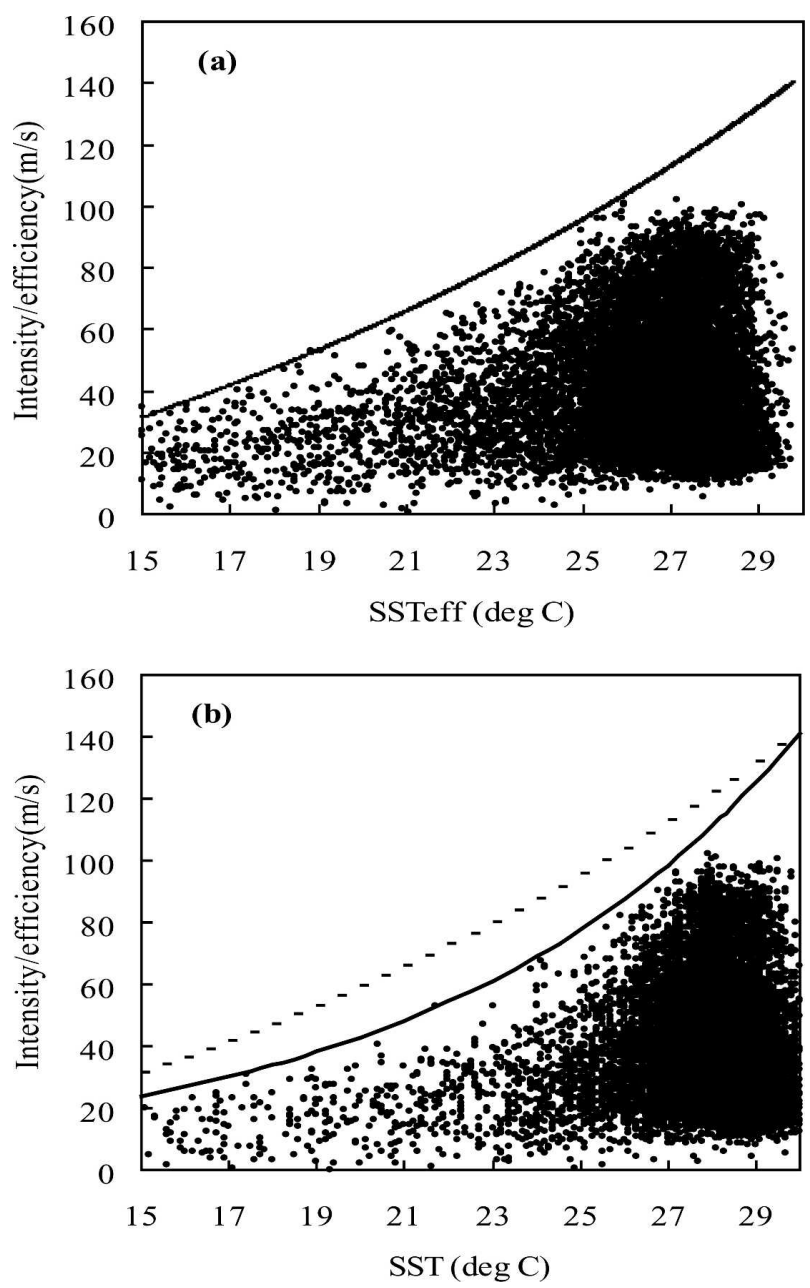

FIG. 13. (a) Scatter diagram of TC intensity $\left(\mathrm{m} \mathrm{s}^{-1}\right)$ divided by the thermodynamic efficiency vs the effective SST $\left({ }^{\circ} \mathrm{C}\right)$ (dots) together with the new empirical MPI (curve). (b) Same as (a) but with the attenuation factor $\eta=1$. Two curves in (b) are the SST-determined empirical MPI with thermodynamic efficiency taken into account (solid curve) and the new MPI with the dynamical control omitted (dashed curve).

from Fig. 14 and Table 6, 54.4\% (14.1\%) of the storms reached $50 \%(80 \%)$ of their new MPI, while only $48.7 \%(8.4 \%)$ and $49.8 \%(9.9 \%)$ reached $50 \%(80 \%)$ of their SST-determined MPI and the MPI modified to include the effect of the thermodynamic efficiency, respectively. Therefore, the estimation of TC maximum intensity is significantly improved due to the inclusion of the dynamical control in the new MPI.

\section{Conclusions}

In this study, based on the best-track TC data, Reynolds SST, and NCEP-NCAR reanalysis during 19812003, we have analyzed the effects of translational
Cumulative distribution of relative intensity

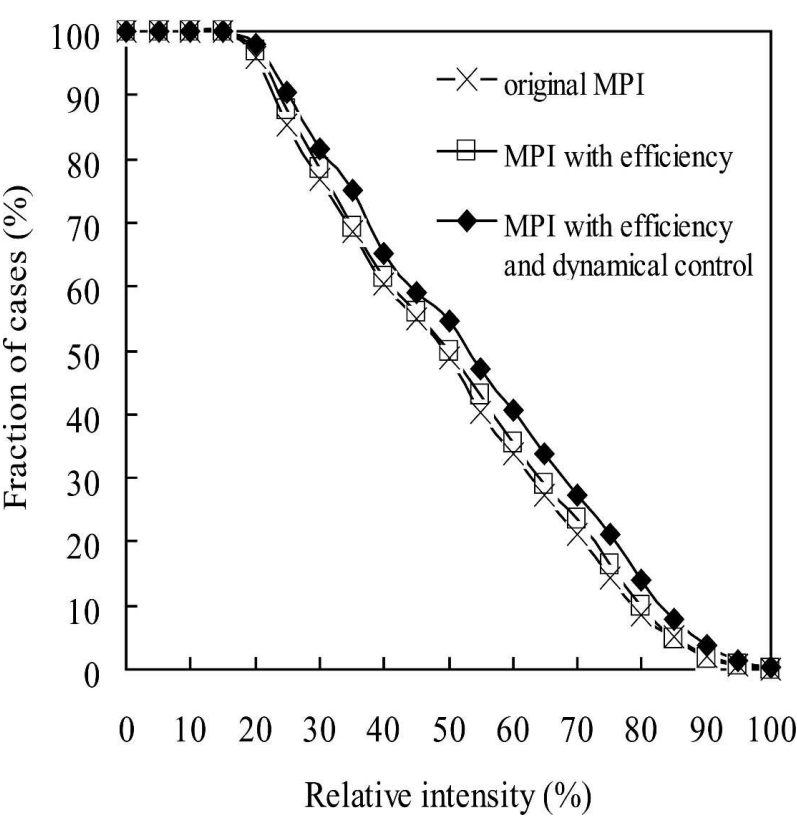

FIG. 14. Cumulative distributions of relative peak intensity from the original SST-determined MPI, the modified MPI with the thermodynamic efficiency included, and the new MPI incorporating environmental dynamical control for the western North Pacific during 1981-2003.

speed and vertical shear on TC intensification, intensity, and the lifetime peak intensity in the western North Pacific. In general, both the fast translation and strong vertical shear are negative to both TC intensification and intensity. Both the very intense TCs and the TCs with rapid intensification rates are found only to occur in a narrow range of translational speeds between 3 and $8 \mathrm{~m} \mathrm{~s}^{-1}$. It is suggested that if a TC moves too slow, cooling induced by turbulent mixing generated by the surface wind stress curl under the TC will disrupt the intensification (Schade and Emanuel 1999; Schade 2000), while if it moves too fast the resulting asymmetric structure will also inhibit intensification (Peng et al. 1999). The overwhelming majority of western North Pacific TCs reach their lifetime peak intensity just prior to recurvature when their environmental steering flow and vertical shear are both weak, consistent with the findings of Evans and McKinley (1998). A robust feature is that few TCs intensified when they moved faster than $15 \mathrm{~m} \mathrm{~s}^{-1}$, especially for storms reaching the typhoon intensity (with maximum sustained surface winds larger than $32 \mathrm{~m} \mathrm{~s}^{-1}$ ).

Although vertical shear is claimed from time to time to be a major limiting factor to TC genesis and intensification, very strong TCs can survive in quite strong 
TABLE 6. Cumulative number of observations and cumulative percentage with relative intensity.

\begin{tabular}{|c|c|c|c|c|c|c|}
\hline \multirow{2}{*}{$\begin{array}{c}\text { Relative } \\
\text { intensity } \\
(\%)\end{array}$} & \multicolumn{2}{|c|}{ Original MPI } & \multicolumn{2}{|c|}{ MPI with efficiency } & \multicolumn{2}{|c|}{$\begin{array}{l}\text { MPI with efficiency and } \\
\text { dynamical control }\end{array}$} \\
\hline & $\begin{array}{l}\text { Cumulative No. } \\
\text { of obs }\end{array}$ & $\begin{array}{c}\text { Cumulative } \\
\text { percentage }(\%)\end{array}$ & $\begin{array}{l}\text { Cumulative No. } \\
\text { of obs }\end{array}$ & $\begin{array}{c}\text { Cumulative } \\
\text { percentage (\%) }\end{array}$ & $\begin{array}{l}\text { Cumulative No. } \\
\text { of obs }\end{array}$ & $\begin{array}{c}\text { Cumulative } \\
\text { percentage (\%) }\end{array}$ \\
\hline 0 & 597 & 100.00 & 597 & 100.00 & 597 & 100.00 \\
\hline 5 & 597 & 100.00 & 597 & 100.00 & 597 & 100.00 \\
\hline 10 & 597 & 100.00 & 597 & 100.00 & 597 & 100.00 \\
\hline 15 & 597 & 100.00 & 597 & 100.00 & 597 & 100.00 \\
\hline 20 & 573 & 95.98 & 578 & 96.82 & 585 & 97.99 \\
\hline 25 & 510 & 85.43 & 523 & 87.60 & 540 & 90.45 \\
\hline 30 & 458 & 76.72 & 469 & 78.56 & 487 & 81.57 \\
\hline 35 & 409 & 68.51 & 414 & 69.35 & 449 & 75.21 \\
\hline 40 & 361 & 60.47 & 367 & 61.47 & 390 & 65.33 \\
\hline 45 & 328 & 54.94 & 334 & 55.95 & 352 & 58.96 \\
\hline 50 & 291 & 48.74 & 297 & 49.75 & 326 & 54.61 \\
\hline 55 & 241 & 40.37 & 256 & 42.88 & 282 & 47.24 \\
\hline 60 & 202 & 33.84 & 211 & 35.34 & 242 & 40.54 \\
\hline 65 & 162 & 27.14 & 174 & 29.15 & 202 & 33.84 \\
\hline 70 & 126 & 21.11 & 140 & 23.45 & 163 & 27.30 \\
\hline 75 & 86 & 14.41 & 97 & 16.25 & 126 & 21.11 \\
\hline 80 & 50 & 8.38 & 59 & 9.88 & 84 & 14.07 \\
\hline 85 & 31 & 5.19 & 28 & 4.69 & 46 & 7.71 \\
\hline 90 & 12 & 2.01 & 10 & 1.68 & 22 & 3.69 \\
\hline 95 & 5 & 0.84 & 5 & 0.84 & 8 & 1.34 \\
\hline 100 & 1 & 0.17 & 0 & 0.00 & 2 & 0.34 \\
\hline
\end{tabular}

vertical shears over the western North Pacific in support of the results of Wang et al. (2004). Zehr (1992) found a threshold vertical shear of $12.5 \mathrm{~m} \mathrm{~s}^{-1}$ above which no TCs could form, we found in this study that few TCs intensified when the vertical shear was larger than $20 \mathrm{~m} \mathrm{~s}^{-1}$ and that most TCs reached their lifetime peak intensity with vertical shear less than $20 \mathrm{~m} \mathrm{~s}^{-1}$. The intensification rate generally increases with decreasing vertical shear while the majority of the weakening storms experience relatively strong vertical shear. Therefore, overall strong vertical shear prohibits rapid intensification and most likely results in the TC weakening, much the same as the fast storm translation.

Based on the observations, a new empirical MPI has been developed, which includes not only the positive contribution of SST and the effect of the thermodynamic efficiency (or outflow temperature) but also the combined negative effect of translational speed and vertical shear as the environmental dynamical control of TC maximum intensity. It is found that the introduction of a thermodynamic efficiency to the SSTdetermined empirical MPI only improves the estimation of TC MPI marginally because the thermodynamic efficiency is closely related to SST. The new empirical MPI with dynamical control provides a more accurate estimation of TC maximum intensity than the original empirical MPI, which is a function of SST only or the one that includes the effect of outflow temperature through the thermodynamic efficiency. The new empirical MPI also provides an approximate, explicit measure of the thermodynamic and environmental dynamical controls of TC maximum intensity, thus improving our understanding of factors controlling TC intensity. Another possible application of the new empirical MPI we have developed is to assess the impact of climate change on TC intensity by global models, which can supposedly provide reliable projection of large-scale features such as the mean flow and vertical shear fields but could not produce the realistic TC intensity due to their limited model resolution.

Note that the MPI only provides an upper limit of TC intensity. There are a large portion of TCs that could not reach their MPI even in favorable environmental conditions. This indicates that other factors also play important roles in limiting TC intensity, such as the approaching landmass, the effect of oceanic cooling due to turbulent mixing, and the internal dynamics of TC themselves, etc. To improve TC intensity forecast, understanding and identifying the major limiting factors are very important. Future studies may pay more attention to the effect of internal dynamics on TC intensity change, as suggested in Wang and Wu (2004). In particular, TC intensity change is largely determined by how the inner-core dynamics respond to environmental forcing and the underlying ocean cooling. Similar analyses can be extended to other TC basins to examine 
whether similar empirical MPIs incorporating dynamical control can be constructed.

Acknowledgments. The authors are grateful to Dr. C. Landsea for his constructive review comments. This study has been supported by the National Science Foundation under Grant ATM-0427128 and by the U.S. Office of Naval Research under Grant N0014-021-0532. Zeng was partly supported by the National Natural Science Foundation of China under Grants 40575030 and 40275018. Additional support has been provided by JAMSTEC (Japan) through its sponsorship of the International Pacific Research Center at the University of Hawaii at Manoa.

\section{REFERENCES}

Bender, M. A., I. Ginis, and Y. Kurihara, 1993: Numerical simulations of tropical cyclone-ocean interaction with a high resolution coupled model. J. Geophys. Res., 98, 23 245-23 263.

Bister, M., and K. A. Emanuel, 1998: Dissipative heating and hurricane intensity. Meteor. Atmos. Phys., 50, 233-240.

Camp, J. P., and M. T. Montgomery, 2001: Hurricane maximum intensity: Past and present. Mon. Wea. Rev., 129, 1704-1717.

DeMaria, M., 1996: The effect of vertical wind shear on tropical cyclone intensity change. J. Atmos. Sci., 53, 2076-2087.

— diction scheme (SHIPS) for the Atlantic basin. Wea. Forecasting, 9, 209-220.

— intensity of Atlantic tropical cyclones. J. Climate, 7, 13251334.

— Prediction Scheme (SHIPS) for the Atlantic and eastern North Pacific basins. Wea. Forecasting, 14, 326-337.

_- M. Mainelli, L. K. Shay, J. A. Knaff, and J. Kaplan, 2005: Further improvements to the Statistical Hurricane Intensity Prediction Scheme (SHIPS). Wea. Forecasting, 20, 531-543.

Dengler, K., and D. Keyser, 2000: Intensification of tropical cyclone-like vortices in uniform zonal background flows. Quart. J. Roy. Meteor. Soc., 126, 549-568.

Elsberry, R. L., and R. Jeffries, 1996: Vertical wind shear influences on tropical cyclone formation and intensification during TCM-92 and TCM-93. Mon. Wea. Rev., 124, 1374-1387.

Emanuel, K. A., 1986: An air-sea interaction for tropical cyclones. Part I: Steady-state maintenance. J. Atmos. Sci., 43, 585-604.

, 1988: The maximum intensity of hurricanes. J. Atmos. Sci., 45, 1143-1155.

_ 1991: The theory of hurricanes. Annu. Rev. Fluid Mech., 23, 179-196.

_- 1995: Sensitivity of tropical cyclones to surface exchange coefficients and a revised steady-state model incorporating eye dynamics. J. Atmos. Sci., 52, 3969-3976.

- 1999: Thermodynamic control of hurricane intensity. $\mathrm{Na}$ ture, 401, 665-669.

_ 2000: A statistical analysis of hurricane intensity. Mon. Wea. Rev., 128, 1139-1152.

— C. Des Autels, C. Holloway, and R. Korty, 2004: Environ- mental control of tropical cyclone intensity. J. Atmos. Sci., 61, 843-858.

Evans, J. L., and K. McKinley, 1998: Relative timing of tropical storm lifetime maximum intensity and track recurvature. $\mathrm{Me}$ teor. Atmos. Phys., 65, 241-245.

Frank, W. M., and E. A. Ritchie, 2001: Effects of vertical wind shear on the intensity and structure of numerically simulated hurricanes. Mon. Wea. Rev., 129, 2249-2269.

Franklin, J. L., S. J. Lord, S. E. Feuer, and F. D. Marks, 1993: The kinematic structure of Hurricane Gloria (1985) determined from nested analyses of dropwindsonde and Doppler radar data. Mon. Wea. Rev., 121, 2433-2451.

Free, M., M. Bister, and K. Emanuel, 2004: Potential intensity of tropical cyclones: Comparison of results from radiosonde and reanalysis data. J. Climate, 17, 1722-1727.

Gray, M. W., 1968: Global view of the origin of tropical disturbances and storms. Mon. Wea. Rev., 96, 669-700.

, 1978: Hurricanes: Their formation, structure and likely role in the tropical circulation. Meteorology over the Tropical Oceans, D. B. Shaw, Ed., Roy. Meteor. Soc., 127-139.

Hanley, D., J. Molinari, and D. Keyser, 2001: A composite study of the interaction between tropical cyclones and uppertropospheric troughs. Mon. Wea. Rev., 129, 2570-2584.

Henderson-Sellers, A., and Coauthors, 1998: Tropical cyclones and global climate change: A post-IPCC assessment.. Bull. Amer. Meteor. Soc., 79, 19-38.

Holland, G. J., 1997: The maximum potential intensity of tropical cyclones. J. Atmos. Sci., 54, 2519-2541.

Kalnay, E., and Coauthors, 1996: The NCEP/NCAR 40-Year Reanalysis Project. Bull. Amer. Meteor. Soc., 77, 437-471.

Kepert, J. D., 2001: The dynamics of boundary layer jets within the tropical cyclone core. Part I: Linear theory. J. Atmos. Sci., 58, 2469-2484.

— , and Y. Wang, 2001: The dynamics of boundary layer jets within the tropical cyclone core. Part II: Nonlinear enhancement. J. Atmos. Sci., 58, 2485-2501.

Knaff, J. A., C. R. Sampson, and M. DeMaria, 2005: An operational statistical typhoon intensity prediction scheme for the western North Pacific. Wea. Forecasting, 20, 688-699.

Malkus, J. S., and H. Riehl, 1960: On the dynamics and energy transformation in steady-state hurricanes. Tellus, 12, 1-20.

McBride, J. L., and R. Zehr, 1981: Observational analysis of tropical cyclone formation. Part II: Comparison of non-developing versus developing systems. J. Atmos. Sci., 38, 1132-1151.

Merrill, R. T., 1988: Environmental influences on hurricane intensification. J. Atmos. Sci., 45, 1678-1687.

Miller, B. I., 1958: On the maximum intensity of hurricanes. $J$. Meteor., 15, 184-195.

Möller, J. D., and M. T. Montgomery, 1999: Vortex Rossby waves and hurricane intensification in a barotropic model. J. Atmos. Sci., 56, 1674-1687.

— , and — 2000: Tropical cyclone evolution via potential vorticity anomalies in a three-dimensional balance model. $J$. Atmos. Sci., 57, 3366-3387.

Montgomery, M. T., and R. J. Kallenbach, 1997: A theory for the vortex Rossby waves and its application to spiral bands and intensity changes in hurricanes. Quart. J. Roy. Meteor. Soc., 123, 435-465.

— tively forced vortex Rossby waves in a three-dimensional quasigeostrophic model. J. Atmos. Sci., 55, 3176-3207.

Nong, S., and K. Emanuel, 2003: A numerical study of the genesis 
of concentric eyewalls in hurricanes. Quart. J. Roy. Meteor. Soc., 129, 3323-3338.

Palmén, E., 1948: On the formation and structure of tropical hurricanes. Geophysica, 3, 26-38.

Peng, M. S., B.-F. Jeng, and R. T. Williams, 1999: A numerical study on tropical cyclone intensification. Part I: Beta effect and mean flow effect. J. Atmos. Sci., 56, 1404-1423.

Reynolds, R. W., N. A. Rayner, T. M. Smith, D. C. Stokes, and W. Wang, 2002: An improved in situ and satellite SST analysis for climate. J. Climate, 15, 1609-1625.

Schade, L. R., 2000: Tropical cyclone intensity and sea surface temperature. J. Atmos. Sci., 57, 3122-3130.

—_ and K. A. Emanuel, 1999: The ocean's effect on the intensity of tropical cyclones: Results from a simple coupled atmosphere-ocean model. J. Atmos. Sci., 56, 642-651.

Shapiro, L. J., 1983: The asymmetric boundary layer flow under a translation hurricane. J. Atmos. Sci., 40, 1984-1998.

Titley, D. W., and R. L. Elsberry, 2000: Large intensity changes in tropical cyclones: A case study of supertyphoon Flo during TCM-90. Mon. Wea. Rev., 128, 3556-3573.

Tonkin, H., G. J. Holland, N. Holbrook, and A. HendersonSellers, 2000: An evaluation of thermodynamic estimates of climatological maximum potential tropical cyclone intensity. Mon. Wea. Rev., 128, 746-762.

Wang, Y., 2001: An explicit simulation of tropical cyclones with a triply nested movable mesh primitive equation model: TCM3. Part I: Model description and control experiment. Mon. Wea. Rev., 129, 1370-1394.

__, 2002a: Vortex Rossby waves in a numerically simulated tropical cyclone. Part I: Overall structure, potential vorticity, and kinetic energy budgets. J. Atmos. Sci., 59, 1213-1238.

2002b: Vortex Rossby waves in a numerically simulated tropical cyclone. Part II: The role in tropical cyclone structure and intensity changes. J. Atmos. Sci., 59, 1239-1262.

_ 2002c: An explicit simulation of tropical cyclones with a triply nested movable mesh primitive equation model:
TCM3. Part II: Model refinements and sensitivity to cloud microphysics parameterization. Mon. Wea. Rev., 130, 30223036.

—, and G. J. Holland, 1995: On the interaction of tropicalcyclone-scale vortices. IV: Baroclinic vortices. Quart. J. Roy. Meteor. Soc., 121, 95-126.

—, and C.-C. Wu, 2004: Current understanding of tropical cyclone structure and intensity changes-A review. Meteor. Atmos. Phys., 87, 257-278.

— M. T. Montgomery, and B. Wang, 2004: How much vertical shear can a tropical cyclone resist? Bull. Amer. Meteor. Soc., 85, 661-662.

Whitney, L. D., and J. S. Hobgood, 1997: The relationship between sea surface temperatures and maximum intensities of tropical cyclones in the eastern North Pacific Ocean. J. Climate, 10, 2921-2930.

Willoughby, H. E., J. A. Clos, and M. G. Shoreibah, 1982: Concentric eyewalls, secondary wind maxima, and the evolution of the hurricane vortex. J. Atmos. Sci., 39, 395-411.

—, H.-L. Jin, S. J. Lord, and J. M. Piotrowicz, 1984: Hurricane structure and evolution as simulated by an axisymmetric, nonhydrostatic numerical model. J. Atmos. Sci., 41, 11691186.

Wong, M. L. M., and J. C. L. Chan, 2004: Tropical cyclone intensity in vertical wind shear. J. Atmos. Sci., 61, 1859-1876.

Wu, C.-C., and H.-J. Cheng, 1999: An observational study of environmental influences on the intensity changes of Typhoons Flo (1990) and Gene (1990). Mon. Wea. Rev., 127, 3003-3031.

Wu, L., and S. A. Braun, 2004: Effects of environmentally induced asymmetries on hurricane intensity: A numerical study. $J$. Atmos. Sci., 61, 3065-3081.

Yang, B., Y. Wang, and B. Wang, 2007: The effect of internally generated inner-core asymmetries on tropical cyclone intensity. J. Atmos. Sci., in press.

Zehr, R. M., 1992: Tropical cyclogenesis in the western north $\mathrm{Pa}-$ cific. NOAA Tech. Rep. NESDIS 61, 181 pp. 